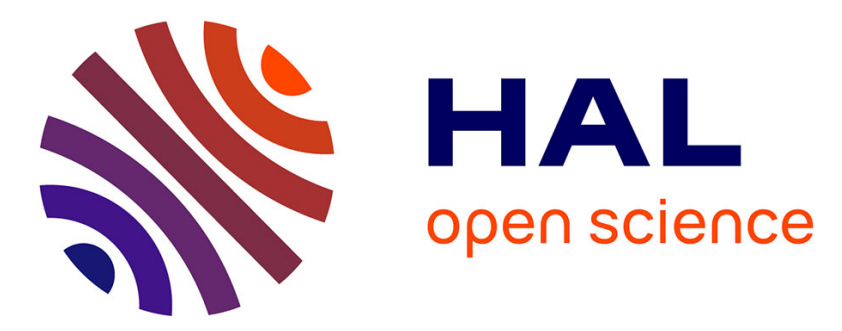

\title{
Sampling the thermal Wigner density via a generalized Langevin dynamics
}

Thomas Plé, Simon Huppert, Fabio Finocchi, Philippe Depondt, Sara Bonella

\section{To cite this version:}

Thomas Plé, Simon Huppert, Fabio Finocchi, Philippe Depondt, Sara Bonella. Sampling the thermal Wigner density via a generalized Langevin dynamics. Journal of Chemical Physics, 2019 , 151 (11), pp.114114. 10.1063/1.5099246 . hal-02298223

\section{HAL Id: hal-02298223 \\ https://hal.sorbonne-universite.fr/hal-02298223}

Submitted on 26 Sep 2019

HAL is a multi-disciplinary open access archive for the deposit and dissemination of scientific research documents, whether they are published or not. The documents may come from teaching and research institutions in France or abroad, or from public or private research centers.
L'archive ouverte pluridisciplinaire HAL, est destinée au dépôt et à la diffusion de documents scientifiques de niveau recherche, publiés ou non, émanant des établissements d'enseignement et de recherche français ou étrangers, des laboratoires publics ou privés. 


\title{
Sampling the thermal Wigner density via a generalized Langevin dynamics
}

\author{
Thomas Plé, ${ }^{1}$ Simon Huppert, ${ }^{1}$ Fabio Finocchi, ${ }^{1}$ Philippe Depondt, ${ }^{1}$ and Sara Bonella ${ }^{2}$ \\ 1) Sorbonne Université, CNRS, Institut des NanoSciences de Paris, INSP, 4 place Jussieu, F-75005 Paris, \\ France \\ ${ }^{2)}$ CECAM Centre Européen de Calcul Atomique et Moléculaire, École Polytechnique Fédérale de Lausanne, \\ Batochimie, Avenue Forel 2, 1015 Lausanne, Switzerland
}

(Dated:)

The Wigner thermal density is a function of considerable interest in the area of approximate (linearized or semiclassical) quantum dynamics where it is employed to generate initial conditions for the propagation of appropriate sets of classical trajectories. In this paper, we propose an original approach to compute the Wigner density, based on a generalized Langevin equation. The stochastic dynamics is non-trivial in that it contains a coordinate-dependent friction coefficient and a generalized force that couples momenta and coordinates. These quantities are, in general, not known analytically and have to be estimated via auxiliary calculations. The performance of the new sampling scheme is tested on standard model systems with highly non classical features such as relevant zero point energy effects, correlation between momenta and coordinates, and negative parts of the Wigner density. In its current brute force implementation, the algorithm, whose convergence can be systematically checked, is accurate and has only limited overhead compared to schemes with similar characteristics. We briefly discuss potential ways to further improve its numerical efficiency.

\section{INTRODUCTION}

Nuclear quantum effects are relevant to describe processes of experimental significance occurring at low temperature or high pressure, and even surprisingly close to ambient conditions, as in the case of reactions involving proton transfer. The growing interest in quantum computing, and, in general, in engineering of materials approaching scales where classical mechanics fails, further motivates work in this area. From a computational point of view, for distinguishable particles (and bosonic systems), the evaluation of time-independent statistical properties has been essentially solved within the framework of path integrals by mapping the quantum thermal density into a Boltzmann-like function for an isomorphic classical system ${ }^{1-4}$. In this isomorphism, each quantum particle is represented via a set of replicas, or beads, governed by a purely coordinate dependent potential. The representation is exact in the limit of an infinite number of replicas and its numerical convergence can be systematically tested as a function of this number. Classical techniques can then be applied to sample the density with a cost that increases, at most, linearly with the number of replicas compared to the classical case. Although path integrals do provide the reference method, their numerical cost can become problematic if not prohibitive for systems requiring large number of replicas for convergence, especially when first principles interactions are needed. This motivated the development of alternative approaches, in particular the family of the Quantum Thermal Bath (QTB) schemes, that reproduce statistical quantum properties such as zero point energy via a Langevin equation with colored noise ${ }^{5,6}$. Although not rigorously derivable, these equations can be integrated at a cost comparable to that of classical calculations and QTB has proved useful for several interesting systems $^{7-10}$.
While path integrals play a prominent role in sampling the quantum thermal density, considerable attention has also been paid to the Wigner formulation of quantum mechanics ${ }^{11}$. This formalism is based on a correspondence between quantum operators and functions in a (generalized) phase space. The Wigner transform of the quantum operator $\hat{A}$ is defined as

$$
A_{w}(q, p)=\int d \Delta e^{\frac{i p}{\hbar} \Delta}\left\langle q-\frac{\Delta}{2}|\hat{A}| q+\frac{\Delta}{2}\right\rangle
$$

Here and in most of the following we use one-dimensional notation, the full dimensional expressions for our developments are presented in appendix D. Wigner's formalism enables to express quantum averages in a form with striking analogies with the classical case. Timeindependent thermal quantum averages, in particular, can in fact be written as

$$
\langle\hat{A}\rangle=\int d q d p W(q, p) A_{w}(q, p)
$$

where we have introduced the Wigner thermal density:

$$
W(q, p)=\frac{1}{2 \pi \hbar Q} \int \mathrm{d} \Delta e^{\frac{i p}{\hbar} \Delta}\left\langle q-\frac{\Delta}{2}\left|e^{-\beta \hat{H}}\right| q+\frac{\Delta}{2}\right\rangle
$$

with $Q$ the partition function, $\beta=1 / k_{B} T$ the inverse thermal energy and $\hat{H}=\hat{p}^{2} / 2 m+V(\hat{q})$ the Hamiltonian of the system of mass $m$. More generally, Wigner's formulation of quantum mechanics provides, a complete framework to compute static and time-dependent averages for quantum systems. However, even for static properties, its practical use presents a notable difficulty compared to path integrals. In fact, the Wigner density defined in eq.(3) is, in general, not positive, which prevents its immediate interpretation as a probability density. Furthermore, since the analytic form of this function is generally unknown, one must rely on numerical methods to estimate it. This calculation, however, is highly 
non-trivial because brute force schemes suffer from a numerical sign problem due to the oscillating phase factor $e^{\frac{i p}{\hbar} \Delta}$, which becomes rapidly unmanageable for generic high-dimensional systems. In spite of these difficulties, the Wigner density remains a key quantity, in particular, in the area of linearized approximations of quantum time-correlation functions ${ }^{12-17}$. In fact, in these methods (and in semiclassical dynamics, see ref. 18 and references therein), the Wigner density appears naturally and its modulus is used to sample the set of initial conditions that are then propagated classically to compute the timeevolved observables. Of particular interest for this work is a set of papers focusing on path integral based Liouville dynamics to compute time-correlation functions in the so-called initial value representation or, more recently, using a Langevin evolution ${ }^{19-23}$. Several strategies have been suggested to sample a positive approximation of eq. (3). Methods for multidimensional systems often rely on a local harmonic approximation ${ }^{14}$ of the potential, modified if necessary via an ansatz to treat imaginary frequencies ${ }^{24}$. Alternatively, Poulsen et al. ${ }^{25}$, combined the variational harmonic frequency path integral representation of Feynman and Kleinert ${ }^{26}$ with the centroid quasi density formalism ${ }^{27}$ to obtain an approximate form of the Wigner density calculable via a relatively simple iterative scheme $^{25}$. Other approaches compute the Wigner function by solving Bloch?s equation in phase space via the propagation of Gaussian packets in imaginary time ${ }^{28,29}$. An other study suggested employing QTB trajectories to approximate the Wigner density ${ }^{30}$. These methods have proved effective on given applications on model and condensed phase systems. However, they all consider only the positive part of the Wigner function and introduce approximations that often cannot be assessed systematically from the theory or in the implementations. Recently, an alternative approach was proposed ${ }^{31}$, relying on an Edgeworth expansion (see Section II) to control the phase factor in eq.(3). This approach is based on a generalized Monte Carlo sampling scheme essentially as efficient as the most common alternatives. The method was shown to capture interesting quantum effects, such as the correct correlations between different degrees of freedom (including coordinate-momentum and momentummomentum correlations) and to provide reliable indication of the existence of negative parts of the Wigner density. Furthermore, the numerical convergence - or failure to converge - of the method can be systematically tested. In this paper, we exploit the Edgeworth expansion of the Wigner density to propose an alternative method based on a generalized Langevin dynamics. This dynamics, that we shall refer to as Wigner-Langevin dynamics (WiLD), rigorously samples the Edgeworth expansion ${ }^{32}$ of the Wigner density up to third order. It can also be used to calculate the successive terms in the Edgeworth series that asymptotically converges to the exact result. It shares with the Monte Carlo scheme proposed in ref. 31 the feature that its numerical convergence can be systematically tested. The method, in its current form, has a slightly higher numerical cost than the previously proposed generalized Monte Carlo scheme, but some further developments indicated in this work (section III B) might improve its efficiency. This approach may also provide an interesting starting point for examining other approximate schemes (e.g. QTB) from a different point of view. Furthermore, in contrast with the existing Monte Carlo scheme, the WiLD can be used also to directly access time-dependent properties of the system. Although in this work we limit ourselves to testing it as a sampling tool for the Wigner density, the method is introduced as a first step in this direction.

The paper is organized as follows. In Section II we start by deriving a convenient Edgeworth expansion of the Wigner density. We then introduce and discuss the new generalized Langevin dynamics employed in all calculations reported in this work and analyze its classical and harmonic limit. As in the case of the generalized Monte Carlo procedure, some of the quantities appearing in the Wigner-Langevin equations of motion are not known analytically but can be computed via auxiliary calculations. After providing estimators for all the quantities that appear in the WiLD equations, we discuss in section III the algorithm used to propagate the stochastic trajectory and highlight its non-trivial aspects. Section IV illustrates the performance of the method on a set of model systems based on those used in ref. 31. To focus the attention on the key elements of the Wigner-Langevin dynamics, its derivation, the specific algorithm used in the auxiliary calculations, the multidimensional version of our equations, and all necessary details for practical implementation of the method appear in the appendices and in supplementary information.

\section{THEORY}

\section{A. Edgeworth expansion}

Let us begin by rewriting eq.(3) as

$$
\begin{aligned}
W(q, p)= & \frac{1}{2 \pi \hbar Q} e^{-\beta U(q)} e^{-\kappa_{2}(q) \frac{p^{2}}{2 \hbar^{2}}} \\
& \times \int \mathrm{d} \Delta e^{\frac{i p}{\hbar} \Delta+\kappa_{2}(q) \frac{p^{2}}{2 \hbar^{2}}} \rho_{c}(\Delta \mid q)
\end{aligned}
$$

where

$$
\begin{gathered}
e^{-\beta U(q)}=\int \mathrm{d} \Delta\left\langle q-\frac{\Delta}{2}\left|e^{-\beta \hat{H}}\right| q+\frac{\Delta}{2}\right\rangle, \\
\rho_{c}(\Delta \mid q)=\frac{\left\langle q-\frac{\Delta}{2}\left|e^{-\beta \hat{H}}\right| q+\frac{\Delta}{2}\right\rangle}{\int \mathrm{d} \Delta\left\langle q-\frac{\Delta}{2}\left|e^{-\beta \hat{H}}\right| q+\frac{\Delta}{2}\right\rangle}
\end{gathered}
$$

and $\kappa_{2}(q)$ is, so far, an arbitrary function of position. 
Expanding $e^{\frac{i p}{\hbar} \Delta+\frac{\kappa_{2} p^{2}}{2 \hbar^{2}}}$ as a power series with respect to $p$, and choosing $\kappa_{2}$ as the second cumulant of the density $\rho_{c}$,

$$
\kappa_{2}(q)=\int \mathrm{d} \Delta \Delta^{2} \rho_{\mathrm{c}}(\Delta \mid q)=\left\langle\Delta^{2}\right\rangle_{\rho_{c}}
$$

yields the so-called Edgeworth expansion:

$$
\begin{aligned}
& W(q, p)=\frac{1}{2 \pi \hbar Q} e^{-\beta U(q)} e^{-\kappa_{2}(q) \frac{p^{2}}{2 \hbar^{2}}} \\
& \times \underbrace{\left[1+\frac{\kappa_{4}(q)}{4 !}\left(\frac{i p}{\hbar}\right)^{4}+\frac{\kappa_{6}(q)}{6 !}\left(\frac{i p}{\hbar}\right)^{6}+\cdots\right]}_{\mathcal{C}_{E W}(q, p)}
\end{aligned}
$$

where $\kappa_{n}(q)$ is the $n$-th order cumulant of $\rho_{c}$, for example, $\kappa_{4}(q)=\left\langle\Delta^{4}\right\rangle_{\rho_{c}}-3\left\langle\Delta^{2}\right\rangle_{\rho_{c}}^{2}$. In equation (8), $W(q, p)$ is written as a product of a positive function times the Edgeworth factor $\mathcal{C}_{E W}(q, p)$, that takes the form of an expansion in powers of $p$. The coefficients of this expansion, the cumulants of $\rho_{c}$, can be computed numerically without facing a sign problem. Note that in the Edgeworth factor $\mathcal{C}_{E W}(q, p)$, odd orders vanish by symmetry of $\rho_{c}$, while the second order term cancels by choice of $\kappa_{2}(q)$. The expansion can take negative values and converges asymptotically towards the exact Wigner density.

In the following, we will indicate as EWn the Edgeworth approximation to the Wigner density obtained by including terms up to order $n$. Therefore EW0 designates the positive exponential factor, while, for instance, EW4 indicates that terms up to $p^{4}$ in $C_{E W}(q, p)$ have been taken into account.

The formal properties of this type of expansion are known $^{32,33}$. In particular, we observe (see IV B) that the difference between properties computed from truncation at two successive orders decreases at first - as in standard convergence - then starts growing again, and eventually diverges. The closest agreement with the reference (numerically exact) results is obtained as this difference reaches its minimum. Although further tests are necessary, we tentatively propose to use this observation as a tool to identify the best Edgeworth approximation of the Wigner density and of observables in the absence of reference calculations. Of course, in practice the quality of the convergence may vary depending on the specific problem, but so far experience on relevant model systems shows that only few terms are needed to reach satisfactory convergence ${ }^{31}$ and that in many cases, even the exponential factor EW0 alone provides a very good approximation to $W(q, p)$. Indeed, choosing $\kappa_{2}(q)$ as the second order cumulant of $\rho_{c}(\Delta \mid q)$ ensures that all terms proportional to the momentum in $\mathcal{C}_{E W}(q, p)$ vanish for Gaussian distributions. In particular, this is the case in the classical limit, as discussed in appendix B. Our calculations also indicate that deviations from this form are not substantial for the model systems considered in this paper with the notable exception of the quartic potential at low temperature. The following subsections describe the MD scheme that we use to sample the positive phase space distribution EW0. The full Wigner density can then be reconstructed by reweighting each of the sampled phase space points with the factor $\mathcal{C}_{E W}(q, p)$, including as many terms as necessary. In contrast with ref. 31, where the Edgeworth expansion was formulated in a path-integral extended variable space, eq. (8) only involves the physical variables $p$ and $q$. This formulation is therefore simpler and more appropriate to derive generalized Langevin equations of motion (path integral developments only appear in the auxiliary calculation presented in paragraph II C).

\section{B. Langevin equations of motion}

Equation (8) can be conveniently expressed as:

$$
W(q, p)=\frac{1}{2 \pi \hbar Q} e^{-\beta H_{\text {eff }}(q, p)} \times \mathcal{C}_{E W}(q, p)
$$

Here, we defined the effective Hamiltonian as:

$$
H_{\mathrm{eff}}(q, p)=\frac{\kappa_{2}(q)}{\lambda^{2}} \frac{p^{2}}{2 m}+U(q)
$$

where $\lambda=\sqrt{\hbar^{2} \beta / m}$ is the thermal de Broglie wavelength and $U(q)$ is the effective potential implicitely defined in equation (5). With this definition, the positive approximation for $W(q, p)$ takes the form of a modified Boltzmann distribution in which a dimensionless position-dependent factor $\kappa_{2}(q) / \lambda^{2}$ multiplies the standard classical kinetic energy, and the physical potential $V(q)$ is replaced by $U(q)$. The modified Boltzmann distribution $e^{-\beta H_{\text {eff }}(q, p)}$ can be sampled using an appropriate numerical scheme, such as the Monte Carlo procedure introduced in ref. 31. In this work, we propose instead a sampling method based on a suitable generalization of the Langevin equation, the Wigner-Langevin dynamics (WiLD), whose formal derivation via the Fokker-Planck equation is provided in appendix A 1. Below we report and comment on the final form of the WiLD equations of motion:

$$
\left\{\begin{array}{l}
\dot{q}=p / m \\
\dot{p}=F(q, p)-\gamma(q) p+\sigma R(t)
\end{array}\right.
$$

where we introduced the generalized force,

$$
F(q, p)=-\frac{\lambda^{2}}{\kappa_{2}(q)} \frac{\partial U}{\partial q}-\left(\frac{\lambda^{2}}{\kappa_{2}(q)} \beta^{-1}+\frac{p^{2}}{2 m}\right) \frac{1}{\kappa_{2}(q)} \frac{\partial \kappa_{2}}{\partial q},
$$

and the friction coefficient,

$$
\gamma(q)=\frac{\kappa_{2}(q)}{\lambda^{2}} \frac{\beta \sigma^{2}}{2 m}
$$

and $R(t)$ is a normalized Gaussian white noise. The WiLD equations (11) have three main non-standard features. Firstly, the force $F(q, p)$ depends on both position 
and momentum. The unusual dependence on momentum originates from the the position-dependent factor $\kappa_{2}(q) / \lambda^{2}$ that renormalizes the kinetic energy in our effective Hamiltonian (see appendix A 1). Secondly, the friction coefficient $\gamma(q)$ depends on coordinate, and it is not simply a constant related to $\sigma^{2}$ by the fluctuationdissipation theorem as in a standard Langevin dynamics. This fact too is a consequence of the renormalization of the kinetic energy. Third, as discussed in more detail in the Subsection II C, both the force $F(q, p)$ and the friction coefficient $\gamma(q)$ are not known analytically and must be estimated via auxiliary path integral calculations at each time step of the WiLD trajectory. In order to ensure the stability and the accuracy of the evolution, these nonanalytic terms must be estimated with enough precision so that the dynamics is not altered.

We conclude this section by mentioning two relevant cases for the Wigner-Langevin dynamics. Let us first consider the classical limit, $\lambda \rightarrow 0$. As proved in appendix B 1, in this case the effective potential $U(q)$ tends to the physical potential $V(q)$, and the kinetic energy scaling factor $\kappa_{2}(q) / \lambda^{2}$ becomes position-independent and equal to $1 . H_{\text {eff }}$ then tends to the classical Hamiltonian and the WiLD reduces to a standard Langevin dynamics. In this limit the higher-order terms in the Edgeworth expansion also tend to zero, and the Wigner density reduces to the classical Boltzmann probability.

The second interesting case arises when $V(q)$ is a harmonic potential with frequency $\omega$. In this case too (see appendix B 2), the force $F(q, p)$ turns into the classical force (for any temperature), while $\kappa_{2}(q)$ is independent of $q$ and equal to $\lambda^{2} \times \tanh \left(\frac{\beta \hbar \omega}{2}\right) / \frac{\beta \hbar \omega}{2}$. Therefore, for harmonic systems, WiLD combines classical evolution with a fluctuation-dissipation relation such that the sampling occurs at an effective temperature corresponding to the correct quantum harmonic oscillator energy. Since in this case the conditional probability $\rho_{c}(\Delta \mid q)$ is Gaussian, all cumulants of order higher than two are null, and the EW0 approximation to the Wigner density is exact.

Considering these two particular cases, we expect the sampling efficiency of the WiLD to be optimal when the friction coefficient $\gamma(q)$ is of the same order of magnitude as the typical vibrational angular frequencies $\omega$ in the system under study, similarly to the case of a standard Langevin dynamics ${ }^{34}$. In the WiLD approach, however, the value of $\gamma(q)$ is controlled only indirectly through the choice of $\sigma$, see eq. (13).

\section{Path-integrals estimators}

As mentioned in Subsection II B, for general potentials, the Wigner-Langevin force (12) and the friction coefficient (13) need to be estimated via auxiliary calculations. To that end, all non-analytical terms in (12) and (13) are expressed as expectation values over the conditional probability density $\rho_{c}$ defined in equation (14). This probability density is related to the off-diagonal elements of the density matrix $e^{-\beta \hat{H}}$ that can be usefully expressed using path integral formalism. More precisely, we use a symmetric Trotter break-up and introduce auxiliary variables $x_{1}, \ldots, x_{\nu-1}$ to write:

$$
\begin{aligned}
\rho_{c}(\Delta \mid q)=\lim _{\nu \rightarrow \infty} \int \mathrm{d} x_{1} \ldots \mathrm{d} x_{\nu-1} \rho_{\nu}\left(\Delta, x_{1}, \ldots, x_{\nu-1} \mid q\right) \\
\rho_{\nu}\left(\Delta, x_{1}, \ldots, x_{\nu-1} \mid q\right) \propto \exp \left\{-\frac{m \nu}{2 \beta \hbar^{2}}\left[\left(q-\frac{\Delta}{2}-x_{\nu-1}\right)^{2}+\left(q+\frac{\Delta}{2}-x_{1}\right)^{2}+\sum_{\lambda=1}^{\nu-2}\left(x_{\lambda+1}-x_{\lambda}\right)^{2}\right]\right\} \\
\quad \times \exp \left\{-\frac{\beta}{\nu}\left[\frac{1}{2} V\left(q+\frac{\Delta}{2}\right)+\frac{1}{2} V\left(q-\frac{\Delta}{2}\right)+\sum_{\lambda=1}^{\nu-1} V\left(x_{\lambda}\right)\right]\right\}
\end{aligned}
$$

Within this path integral representation, the functions $\kappa_{2}, \frac{\partial U}{\partial q}$ and $\frac{\partial \kappa_{2}}{\partial q}$ appearing in eq. (12) and (13) are expressed as expectation values over the density $\rho_{\nu}$ (see appendix C for derivation):

$$
\begin{aligned}
\kappa_{2}(q) & =\left\langle\Delta^{2}\right\rangle_{\rho_{\nu}\left(\Delta, x_{1}, \ldots, x_{\nu-1} \mid q\right)} \\
\frac{\partial U(q)}{\partial q} & =\frac{1}{\nu}\left\langle\frac{1}{2} \frac{\partial}{\partial q} V\left(q+\frac{\Delta}{2}\right)+\frac{1}{2} \frac{\partial}{\partial q} V\left(q-\frac{\Delta}{2}\right)+\sum_{\lambda=1}^{\nu-1} \frac{\partial}{\partial x_{\lambda}} V\left(x_{\lambda}\right)\right\rangle_{\rho_{\nu}\left(\Delta, x_{1}, \ldots, x_{\nu-1} \mid q\right)} \\
\frac{\partial \kappa_{2}(q)}{\partial q} & =\kappa_{2}(q) \beta \frac{\partial U(q)}{\partial q}-\frac{\beta}{\nu}\left\langle\Delta^{2}\left[\frac{1}{2} \frac{\partial}{\partial q} V\left(q+\frac{\Delta}{2}\right)+\frac{1}{2} \frac{\partial}{\partial q} V\left(q-\frac{\Delta}{2}\right)+\sum_{\lambda=1}^{\nu-1} \frac{\partial}{\partial x_{\lambda}} V\left(x_{\lambda}\right)\right]\right\rangle_{\rho_{\nu}\left(\Delta, x_{1}, \ldots, x_{\nu-1} \mid q\right)}
\end{aligned}
$$

In the following, the multidimensional integration over 
density $\rho_{\nu}$, is performed using an auxiliary Langevin dynamics (see Section III and appendix C). Note that, since the Trotter break-up is applied to off-diagonal elements of the density matrix, $\rho_{\nu}$ is isomorphic to an open chain of harmonically coupled beads as represented in the inset of Fig. 1, in contrast to the closed ring-polymer of standard path-integrals molecular dynamics. The higher order cumulants $\kappa_{n}$ appearing in the Edgeworth expansion (eq. (8)) are also trivially estimated from the path-integral expression of $\rho_{c}$.

The evolution equations described above share some similarities with recent work by Liu et al., notably the Path Integral Liouville Dynamics (PILD) method proposed in Ref. 22. In that work, the Wigner density is expressed, based on the local Gaussian approximation ansatz $^{24}$ (LGA), as the product of a Gaussian distribution for the momenta times the exact position probability density, and equations of motion similar to (11) and (12) are derived. However, in the LGA framework, the covariance matrix in the momentum distribution, the so-called thermal mass, is derived from a - local - second order expansion of the potential. This covariance matrix depends in principle on the coordinates but, in the PILD method, an average position-independent matrix is used for practical applications. This is to be contrasted with our approach in which the full $q$-dependence of $\kappa_{2}(q)$ is retained. In section IV, we show that this dependence becomes important in regions where the curvature of the potential changes significantly. Note also that the Gaussian form of the momentum distribution does not arise, in our scheme, from a local quadratic approximation of the potential (as in the LGA case) but is a consequence of the Edgeworth expansion and that $\kappa_{2}(q)$ is the result of the fully non-local cumulant expression (7).

\section{NUMERICAL METHODS}

\section{A. Integration of the WiLD equations of motion}

The WiLD equations of motion (11) are integrated using a symmetric time-splitting method. We adopt the notations of B. Leimkuhler and C. Matthews in ref. 35 to define different blocks:

$$
\left[\begin{array}{c}
\dot{q} \\
\dot{p}
\end{array}\right]=\underbrace{\left[\begin{array}{c}
p / m \\
0
\end{array}\right]}_{\mathrm{A}}+\underbrace{\left[\begin{array}{c}
0 \\
F_{1}(q)
\end{array}\right]}_{\mathrm{B}_{1}}+\underbrace{\left[\begin{array}{c}
0 \\
F_{2}(q) p^{2}
\end{array}\right]}_{\mathrm{B}_{2}}+\underbrace{\left[\begin{array}{c}
0 \\
-\gamma(q) p+\sigma R(t)
\end{array}\right]}_{\mathrm{O}}
$$

where we have split the generalized force $F(q, p)$ in equation (12) into a purely position-dependent part,

$$
F_{1}(q)=-\frac{\lambda^{2}}{\kappa_{2}(q)}\left[\frac{\partial U}{\partial q}+\beta^{-1} \frac{1}{\kappa_{2}(q)} \frac{\partial \kappa_{2}}{\partial q}\right],
$$

and a $p$-dependent term $p^{2} F_{2}(q)$ with

$$
F_{2}(q)=-\frac{1}{2 m \kappa_{2}(q)} \frac{\partial \kappa_{2}}{\partial q}
$$

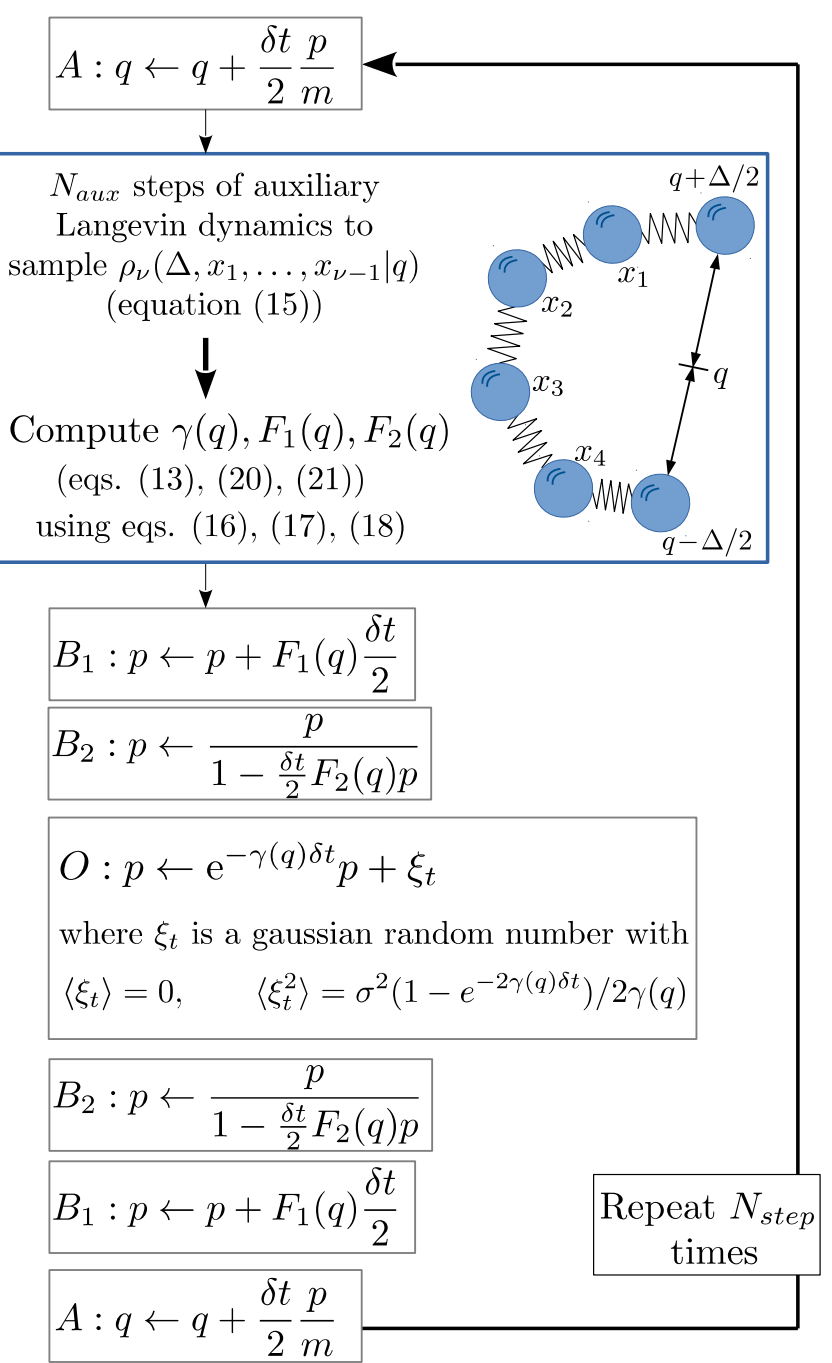

FIG. 1. Flow chart of the WiLD algorithm. The diagram details the integration operations corresponding to the exact propagation of each block in our symmetric time-splitting scheme. The blue box represents the auxiliary path-integral sampling, which is responsible for the major part of the computational load of the method.

corresponding to blocks $\mathrm{B}_{1}$ and $\mathrm{B}_{2}$, respectively. The Liouvillian is decomposed in four components $\mathcal{L}_{A}, \mathcal{L}_{B_{1}}$, $\mathcal{L}_{B_{2}}$ and $\mathcal{L}_{O}$ and the propagator over a time step $\delta t$ is split symmetrically according to:

$$
\begin{array}{r}
e^{\delta t \mathcal{L}}=e^{\frac{\delta t}{2} \mathcal{L}_{A}} e^{\frac{\delta t}{2} \mathcal{L}_{B_{1}}} e^{\frac{\delta t}{2} \mathcal{L}_{B_{2}}} e^{\delta t \mathcal{L}_{O}} e^{\frac{\delta t}{2} \mathcal{L}_{B_{2}}} e^{\frac{\delta t}{2} \mathcal{L}_{B_{1}}} e^{\frac{\delta t}{2} \mathcal{L}_{A}} \\
+\mathcal{O}\left(\delta t^{3}\right)
\end{array}
$$

Each block is then integrated exactly using the expressions provided in the diagram in Fig. 1 ensuring that the discretization error over a time step is of order at least $\delta t^{3}$. This specific time-splitting enables to perform the auxiliary calculation for the factors $\gamma(q), F_{1}(q)$ and $F_{2}(q)$, which constitutes the main numerical load in our 
algorithm, only once per time step.

\section{B. Auxiliary Langevin dynamics}

As shown in Fig. 1, at each time step of a WiLD simulation, an auxiliary path-integral Langevin dynamics is performed in order to sample the probability density $\rho_{\nu}\left(\Delta, x_{1}, \ldots, x_{\nu-1} \mid q\right)$ and estimate the averages defined in equations (16), (17) and (18). The equations of motion for this auxiliary dynamics are derived in appendix C, using standard path integral methods. These equations are integrated using the efficient Path-Integrals OrnsteinUhlenbeck Dynamics (PIOUD) algorithm of ref. 36, that we adapted to the case of an open chain of harmonically coupled beads as explained in the supplementary information. The auxiliary sampling is mainly responsible for the high numerical cost of the method. Indeed, to ensure the stability and the accuracy of the propagation, the variance of the estimators for $\gamma(q), F_{1}(q)$, and particularly $F_{2}(q)$, has to be small.

Two avenues might be pursued in future work to circumvent this requirement. A first strategy might be to compute the averages with high accuracy on a fixed set of points in the $q$ space and then use these values to obtain noiseless estimates in the whole space via interpolation schemes. We have tested this approach with excellent results for the 1-d models presented in this work. Generalizations to higher dimensional systems would be possible adapting methods currently used to fit energy and/or forces via machine learning techniques, based on accurate quantum chemical calculations of the Born-Oppenheimer potential energy surface at a few reference geometries. In this spirit, one could use machine learning approaches, such as artificial neural networks ${ }^{37,38}$ or Gaussian process regression ${ }^{39-41}$, to train a model for the Wigner forces on selected points in position space. A second strategy would consist in adapting to our method the interesting schemes recently proposed to accommodate noisy terms in classical Langevin equations ${ }^{36,42}$. This would allow to relax the current requirement on the accuracy of the estimates and reduce the numerical cost of our dynamics considerably. As a final comment on this point, note that also in the generalized Monte Carlo scheme proposed in ref. 31, a numerical estimate of the "energy" of the system is required, leading to the use of a sampling scheme that accounts for the noise in this estimator. The effective use of these noisy Monte Carlo methods led to an algorithm with the same cost as that of common alternatives (e.g. the Feynman-Kleinert scheme). Based on the tests conducted in this work, using brute force reduction of the variance leads to an algorithm that we estimate two to five times more expensive than its Monte Carlo counterpart. Thus, the developments discussed above should allow to close this gap and establish the WiLD as a competitive method for sampling the Wigner density.

\section{RESULTS}

In this section we present applications of the WiLD to a set of model systems based on those used in ref. 31: one-dimensional Morse and quartic potentials, and a twodimensional model for proton transfer. For each test case, we sample the positive approximation to the Wigner density (EW0) and analyze the effect of the Edgeworth correction terms. The results are compared to the classical Boltzmann distribution (always shown as a dashed gray curve) and to a quantum reference (always shown as a black continuous curve) obtained via a numerical solution of the Schrödinger equation. For all the models presented we used a time step $\delta t=0.1$ fs in the propagation of the WiLD equations and a number of time steps $N_{\text {steps }}$ ranging from $5 \times 10^{6}$ to $15 \times 10^{6}$. Sufficient accuracy for the non-analytical terms is achieved by using a number of auxiliary steps $N_{\text {aux }}$ ranging from 1000 to 2000 . More precisely, we use $N_{t r a j}=10$ parallel independent trajectories of $N_{a u x} / N_{t r a j}$ auxiliary steps each. This generates estimators with uncorrelated noise for the different factors in eqs. (20) and (21), and avoids systematic statistical bias. In particular, the products appearing in these expressions are computed by averaging over all possible unbiased combinations of the $N_{\text {traj }}$ independent estimators. A similar procedure, detailed in appendix $\mathrm{C} 2$, allows eliminating the systematic bias for $1 / \kappa_{2}(q)$ and $1 / \kappa_{2}^{2}(q)$.

The number of beads $\nu$ employed in the auxiliary calculations is indicated in the figure captions.

\section{A. Morse potential}

Let us start by considering the one-dimensional Morse potential:

$$
V(q)=D\left[e^{-2 \alpha q}-2 e^{-\alpha q}\right]+D \zeta(q)
$$

with $D=20 \mathrm{kcal} / \mathrm{mol}, \alpha=2.5 \AA^{-1}$ (same as ref. 31), and for a particle with the mass of a proton. The term $\zeta(q)$ is introduced to prevent dissociation of the system: $\zeta(q)=1$ for $q \leq q_{\max }$ and $\zeta(q)=e^{\eta\left(q-q_{\max }\right)}$ for $q>q_{\max }$ with $q_{\max }=2.5 \AA$ and $\eta=20 \AA^{-1}$.

Figure 2 shows contour plots of the Wigner density at $1200 \mathrm{~K}$ (upper panel) and $300 \mathrm{~K}$ (lower panel), as obtained with the positive approximation EW0 (dasheddotted red curves) and including terms up to 4th-order in the Edgeworth expansion (EW4: dashed green curve). EW0 and EW4 results are almost superimposed in Fig. 2.a and 2.b, indicating convergence of the expansion already at order 0 at both temperatures. In particular, for this system, the Wigner density does not present significant negative regions.

At $1200 \mathrm{~K}$, the thermal energy $k_{B} T$ is of the same order as the zero-point energy for the system, so quantum effects are not very pronounced. The numerically exact 

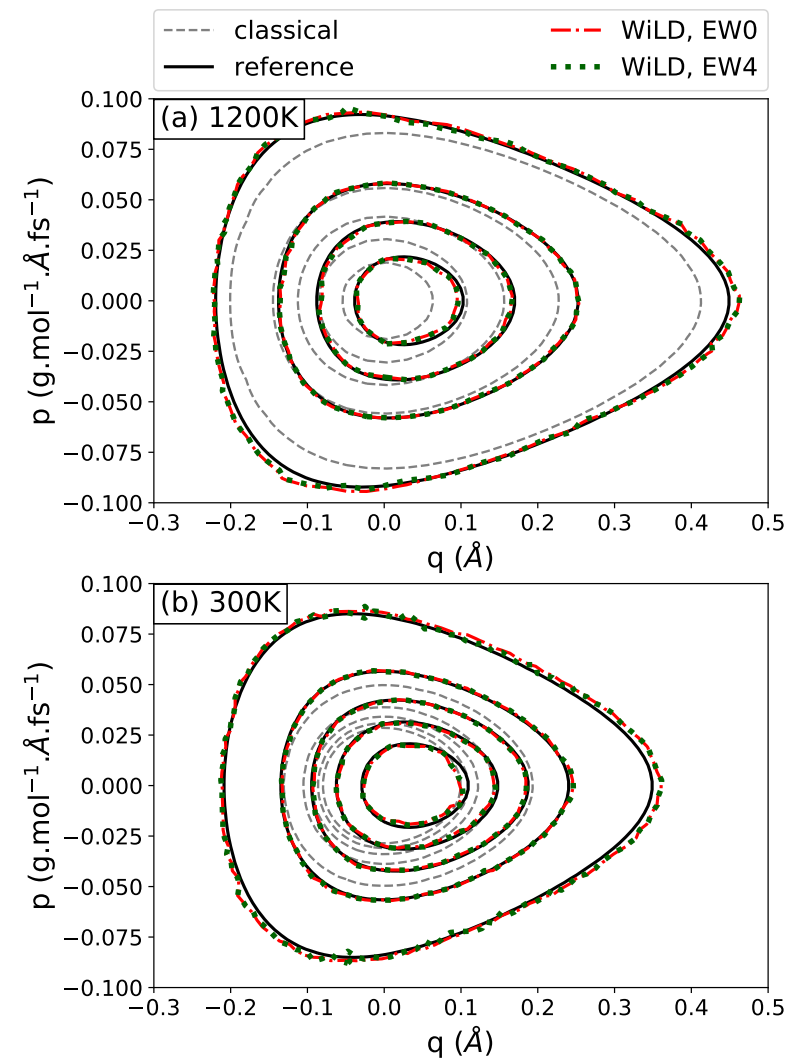

FIG. 2. Contour plots of the Wigner density for the 1D Morse potential. Panel a: $1200 \mathrm{~K}(\nu=8)$. Panel b: $300 \mathrm{~K}(\nu=32)$. Contour levels are 1.5, 10, 20, 30, $40 \mathrm{fs} . \AA^{-2} \cdot(\mathrm{g} / \mathrm{mol})^{-1}$.

Wigner density is similar to the classical Boltzmann distribution, with only a slight broadening and a small shift of the maximum of probability, due to zero-point motion. These effects are well captured by the WiLD sampling.

At $300 \mathrm{~K}, k_{B} T$ becomes significantly smaller than the zero-point energy, and quantum effects are much more significant. The classical Boltzmann distribution is now considerably narrower than the Wigner density, and their respective maxima are clearly shifted from one another. The WiLD sampling matches the quantum result also in this more challenging situation, and the EW0 results are in very good agreement with the correct result. Consistently, even at $300 \mathrm{~K}$, the effect of the Edgeworth correction terms remains very limited: the 4 th order is responsible only for a slight narrowing of the momentum distribution (barely distinguishable on Fig. 2).

It should be stressed that even the simple case of the Morse potential involves some non-trivial quantum effects. In particular it was noted in ref. 31 that the Wigner density presents momentum-position correlations that are completely absent from the separable Boltzmann distribution. Within the WiLD framework, these correlations translate into a position-dependent $\kappa_{2}(q)$. The behaviour of this interesting quantity is reported in Fig. 3.a,
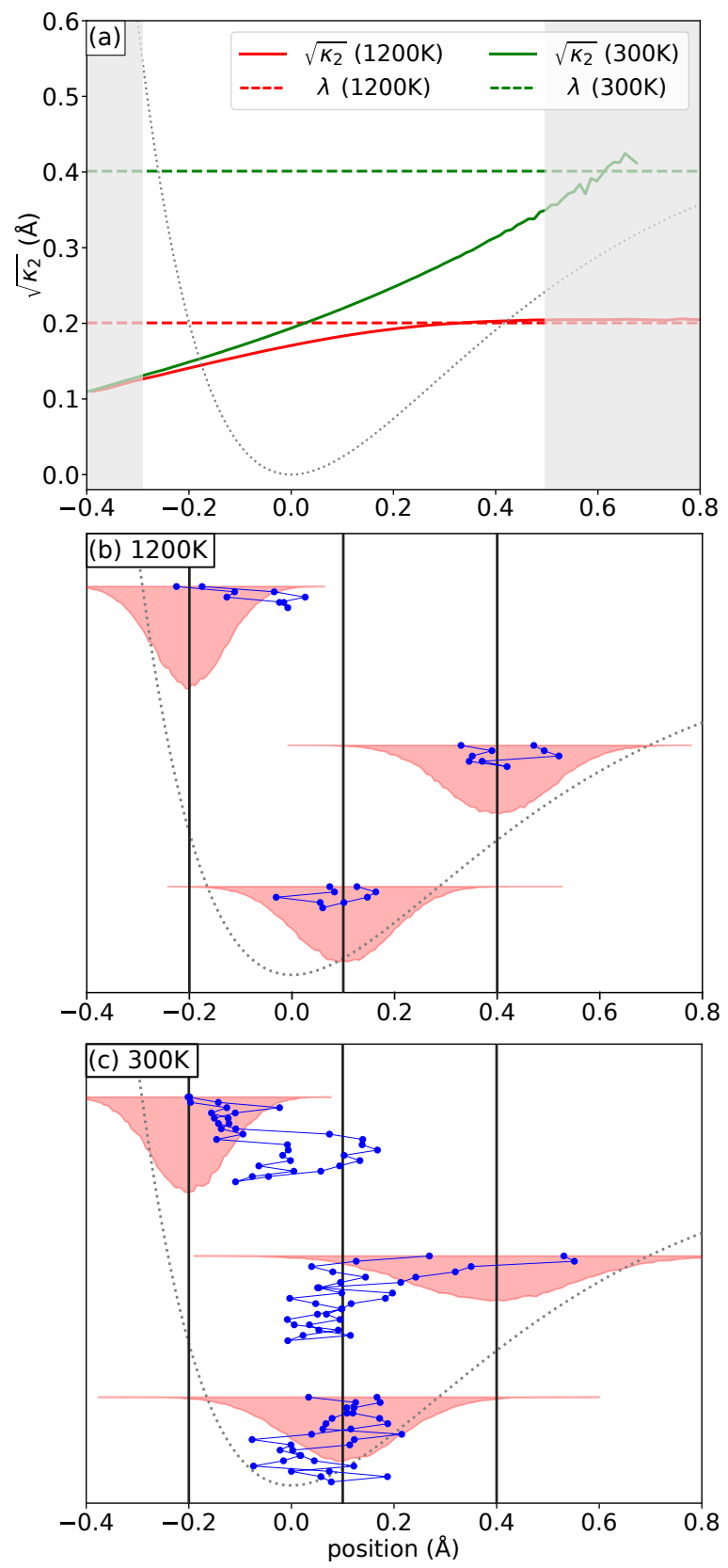

FIG. 3. Panel (a): Plot of $\sqrt{\kappa_{2}(q)}$ at $1200 \mathrm{~K}$ (red) and $300 \mathrm{~K}$ (green). The dashed lines correspond to the classical value, $\lambda$. Panels (b) and (c): Snapshots of typical open chain configurations sampled in the auxiliary Langevin dynamics for the Morse potential at $1200 \mathrm{~K}$ (a) and $300 \mathrm{~K}$ (b). The position of the different beads is indicated with a blue dot at the corresponding value of $x_{\lambda}$ (the position of the dots along the vertical axis is arbitrary and just for visualization purposes). Successive beads in the chain are connected with a blue line. The black vertical lines indicate the value of $q$ for which the auxiliary calculation is performed (i.e. the middle between the two endpoints of the chain): $-0.2 \AA, 0.1 \AA$ and $0.4 \AA$. The figure also shows in red the histogram of the positions of the two endpoints of the open chain, $q-\frac{\Delta}{2}$ and $q+\frac{\Delta}{2}$, sampled during the auxiliary dynamics. The variance of this distribution is directly related to $\kappa_{2}(q)$. On each panel, the potential is superimposed (gray dotted line) with a suitable scale. 
superimposed on the potential $V(q)$ (shown in gray dotted line with an appropriate scale). The figure shows in red and green solid curves the calculated $\kappa_{2}(q)$ at $1200 \mathrm{~K}$ and $300 \mathrm{~K}$, respectively, together with the values of the De Broglie thermal wavelength at the same temperatures (dashed lines). The values of $\kappa_{2}(q)$ are computed by averaging along the WiLD trajectories, therefore the results show increased noise for the values of $q$ corresponding to a low probability. These rarely visited regions are indicated with transparent gray areas. In appendix B 1 we show that, in the classical limit, $\kappa_{2}(q) \rightarrow \lambda$, and that, at a given temperature, larger discrepancies between the classical and quantum result are found in regions of strong curvature of the potential. These results are confirmed by the figure. At the higher temperature, the classical and quantum results (red curves) are almost superimposed for positive $q$, and, in these weakly quantum conditions, appreciable discrepancies only persist close to the repulsive wall of the potential. At the lower temperature, on the other hand, $\kappa_{2}(q)$ differs more significantly from its classical limit. The pronounced dependence on position confirms the existence of relevant momentumposition correlation in the Wigner distribution, a feature not easily captured by alternative methods, and that is correctly accounted for by the WiLD, even at order EW0, as demonstrated by Fig.2. The analysis in appendix B 1 points to the fact that for non-classical systems (i.e. for large $\lambda), \kappa_{2}(q)$ is sensitive to non-local features of the potential. In the semi-classical expression for $\kappa_{2}(q)$, reported in the appendix as an expansion in powers of the thermal wavelength, this effect manifests itself via higher order derivatives of the potential. More generally, the non-locality is apparent in the definition of the average in eq. (7), which depends on the whole potential energy surface and not only on the local curvature at position $q$. This is illustrated also in Fig. 3.b and 3.c, that display snapshots of open chain configurations sampled in the auxiliary Langevin dynamics (see section III and appendix C) at the two temperatures considered. Even at the higher temperature (middle panel), typical configurations of the open chain show rather large displacements from the anchor point $q$ and the central beads of the chain tend to shift towards the minimum of the potential. This is reflected also in the relatively large width of the distribution sampled by the endpoints $q+\Delta / 2$ and $q-\Delta / 2$, whose variance is directly related to $\kappa_{2}(q)$. The non-local nature of this quantity becomes even more evident at $300 \mathrm{~K}$, where the longer chains explore larger portions of the 1D Morse potential and can extend to positions farther from $q$. Similar considerations apply to the other non-analytic terms in the expression (12) of the WiLD force.
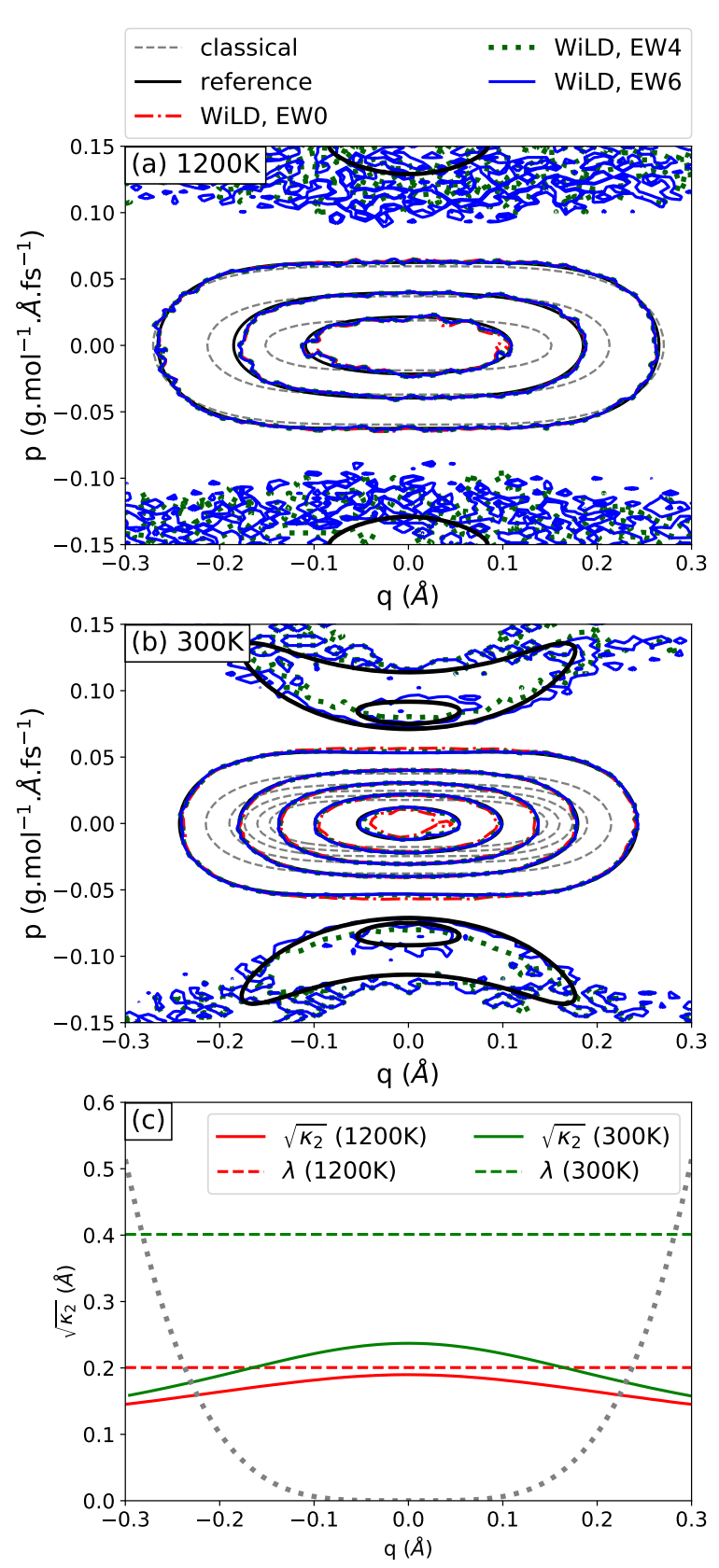

FIG. 4. (a) and (b): Contour plot of the Wigner density for the 1D quartic potential. Panel a: $1200 \mathrm{~K}(\nu=8)$. Panel b: $300 \mathrm{~K}(\nu=32)$. Contour levels are $-0.25,-0.0002,5,15$, $25,35,45 \mathrm{fs} . \AA^{-2} \cdot(\mathrm{g} / \mathrm{mol})^{-1}$. (c): Plot of $\sqrt{\kappa_{2}(q)}$ at $1200 \mathrm{~K}$ (red) and $300 \mathrm{~K}$ (green). The dashed lines correspond to the classical limit, i.e. $\lambda$. The potential is superimposed (gray dotted line) with a suitable scale.

\section{B. Quartic potential}

In this section we consider the case of the onedimensional quartic potential:

$$
V(q)=a \times q^{4}
$$


with $a=800 \AA^{-4} . \mathrm{kcal} / \mathrm{mol}$. The mass of the system was again chosen to be the proton's mass. Figure 4.a and 4.b show the results for the Wigner density at $1200 \mathrm{~K}$ and $300 \mathrm{~K}$, respectively. At $1200 \mathrm{~K}$, the main feature in the quantum and classical densities, the symmetrical oblong peak centered around zero, is quite similar, only the quantum distribution is slightly sharper around $q=0$. The specific shape of the central peak of the Wigner distribution is very well reproduced by the WiLD sampling, at order EW0 (dashed dotted red curve), EW4 (green dotted curve) and EW6 (blue curve) in the Edgeworth expansion. However, even at this relatively high temperature, the numerically exact quantum solution shows symmetrically placed features at large momentum that are absent in the classical and EW0 approximations of the Wigner density. These are negative parts of the Wigner density. They have a very small relative amplitude compared to the main peak (less than $10^{-4}$ ) and are therefore difficult to capture accurately. Encouragingly, however, high order terms in the Edgeworth expansion display some signal in the correct regions (see more below).

As expected, at $300 \mathrm{~K}$, the difference between quantum and classical results becomes more significant. The main feature in the Wigner density is significantly broadened by zero-point motion and, more strikingly, wide regions of negative $W(q, p)$ now appear for large values of $p$. The EW0 approximation of the Wigner density already captures the central (positive) peak of the distribution accurately, with only slight discrepancies with respect to the exact result, that are corrected by the EW4 term. The zero-order approximation also displays significant momentum-position correlations, as evidenced by the variations of $\kappa_{2}(q)$ which deviates appreciably from $\lambda$, as shown in Fig. 4.c. Consistently with the discussion of Fig. 3, this quantity shows particularly marked discrepancies from its classical value in regions of strong curvature of the potential. Moreover, Fig. 4.b and Fig. 4.c show that the high-order terms of the Edgeworth expansion allow describing the negative parts of $W(q, p)$ : at order EW4, negative regions appear in the density but are slightly underestimated, an error that is corrected when the EW6 term is included. For very large momenta, the distribution becomes noisy because the WiLD seldom explores these regions of phase space. In spite of this, the inclusion of higher order terms of the Edgeworth expansion systematically improves the estimate of negative regions of the Wigner density, even when their relative amplitude remains small compared to the positive peak.Here, the (negative) minimum of $W(q, p)$ corresponds to approximately $1 \%$ of its maximum value. Note that the EW4 and EW6 terms in the Edgeworth expansion require longer auxiliary calculations to converge than the force terms used in the EW0 approximation: for example, in figure 4.b, we used $N_{a u x}=5000$ to obtain a satisfactory estimate for the negative parts in the Wigner density, whereas $N_{\text {aux }}=1000$ already yields accurate results for the EW0 approximation. Moreover, the full

\begin{tabular}{|c|c|c|}
\hline & Relative error on $\left\langle p^{2}\right\rangle$ & Relative EWn contribution \\
\hline EW0 & $16 \%$ & \\
\hline EW4 & $4 \%$ & $-11 \%$ \\
\hline EW6 & $-2 \%$ & $-6 \%$ \\
\hline EW8 & $-7 \%$ & $-5 \%$ \\
\hline EW10 & $-12 \%$ & $-6 \%$ \\
\hline EW12 & $-20 \%$ & $-10 \%$ \\
\hline EW14 & $-33 \%$ & $-21 \%$ \\
\hline EW16 & $-61 \%$ & $-71 \%$ \\
\hline
\end{tabular}

TABLE I. Relative error on $\left\langle p^{2}\right\rangle$ compared to exact quantum results for the quartic potential at $300 \mathrm{~K}$. Higher order terms in the Edgeworth expansion are computed by solving the 1D Schrödinger equation and computing $\rho_{c}$ using the wavefunction expression of the thermal density operator. The third column shows the relative contribution of the EWn order term, measured by the relative difference: $\left(\left\langle p^{2}\right\rangle_{E W n}-\left\langle p^{2}\right\rangle_{E W n-2}\right) /\left\langle p^{2}\right\rangle_{E W n}$.

evaluation of the high-order Edgeworth terms becomes cumbersome for systems with many degrees of freedom (see appendix D for details), so that further work may be needed to treat complex systems that display large negative values of $W(q, p)$.

In spite of these numerical difficulties, higher order terms in the Edgeworth expansion can significantly impact the accuracy of calculated physical observables. In table I, we report the relative error in the estimate of the mean square momentum for the quartic potential with respect to the numerically exact reference at $300 \mathrm{~K}$ as a function of the truncation order. The EW0 approximation for the density results in a substantial overestimation of the mean square momentum, which is strongly reduced by including the fourth order term and essentially eliminated at order EW6. Note that the convergence to the exact result is systematically improved at low order (up to order EW6) before degrading slowly at higher orders and finally diverging at very high orders. This is a consequence of the asymptotic character of the convergence of the Edgeworth series. The divergence of the Edgeworth series in that case can be detected by monitoring the contribution of each EWn term on the estimated squared momentum (third column in table IV B): we observe that this contribution first decreases, then reaches a plateau with a relative value around 5\%, before increasing beyond order EW12, as the Edgeworth series begins to diverge. The optimal approximation of the full Wigner density is apparently obtained at order EW6, when this plateau begins, although the general validity of this criterion should be investigated further for other systems with strongly non-Gaussian momentum distributions.

\section{Proton transfer model}

To test the multidimensional generalization of our formalism, we performed WiLD simulations on the following 

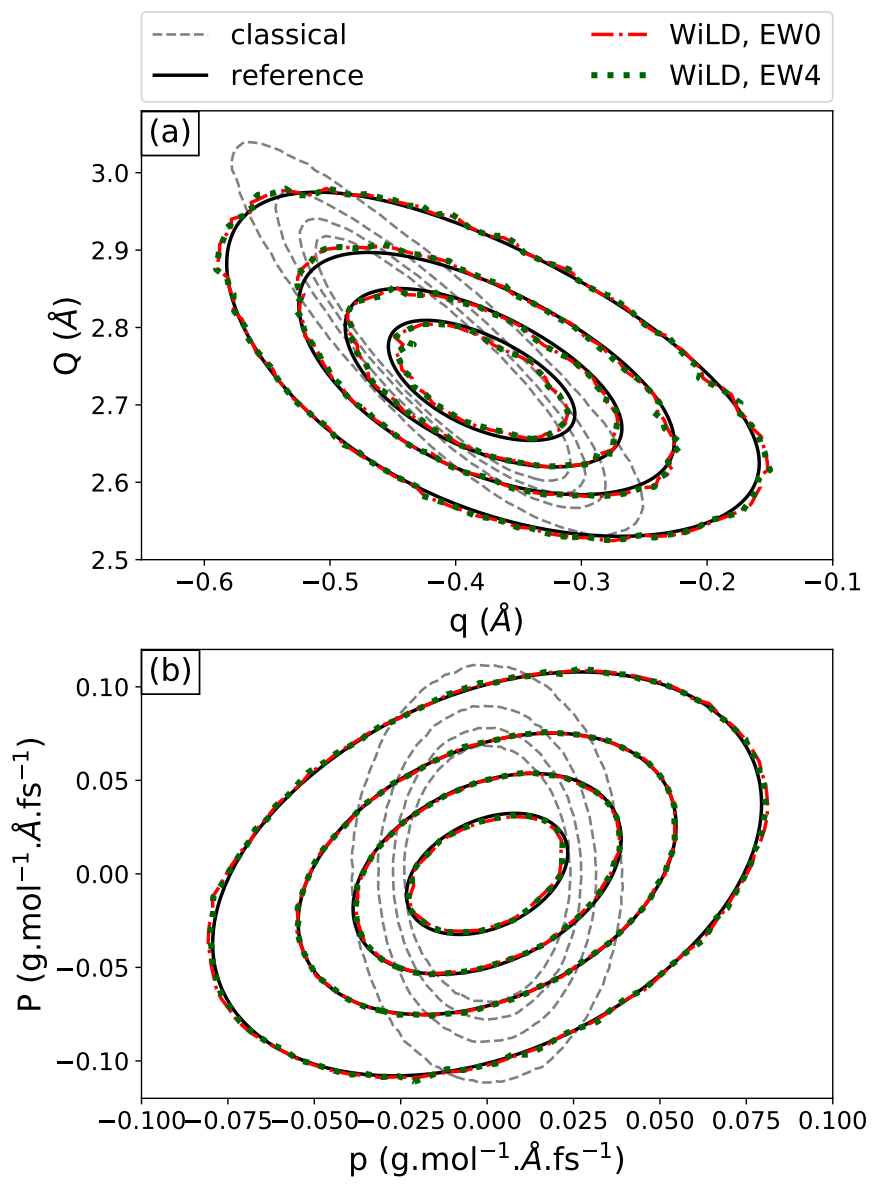

FIG. 5. Contour plot of the position (eq. (26), panel a) and momentum (eq. (27), panel b) probability distributions for the asymmetric hydrogen bond model in equation (25), at $300 \mathrm{~K}$ $(\nu=64)$. The reference is obtained from a direct numerical solution of the two-dimensional Schrödinger equation.

two-dimensional potential ${ }^{31}$ :

$$
\begin{aligned}
V(q, Q)= & D\left[e^{-2 \alpha\left(\frac{Q}{2}+q-d\right)}-2 e^{-\alpha\left(\frac{Q}{2}+q-d\right)}+1\right] \\
& +D \xi^{2}\left[e^{-2 \frac{\alpha}{\xi}\left(\frac{Q}{2}+q-d\right)}-2 e^{-\frac{\alpha}{\xi}\left(\frac{Q}{2}+q-d\right)}\right] \\
& +A e^{-B Q}-\frac{C}{Q^{6}}
\end{aligned}
$$

This model represents an asymmetric A-H-B hydrogen bond, between two different elements $\mathrm{A}$ and $\mathrm{B}$. The coordinate $Q$ is the A-B distance while $q$ is the distance of the proton to the center of the A-B bond. We denote with $P$ and $p$ the associated momenta, respectively. The parameters are chosen similarly to ref. $31: D=60$ $\mathrm{kcal} / \mathrm{mol}, \alpha=2.52 \AA^{-1}, d=0.95 \AA, A=2.32 \times 10^{5}$ $\mathrm{kcal} / \mathrm{mol}, B=3.15 \AA^{-1}, C=2.31 \times 10^{4}(\mathrm{kcal} / \mathrm{mol}) . \AA^{6}$ and the asymmetry factor $\xi=0.707$.

The simulations were performed at 300K. The WiLD equations of motion for multidimensional systems are de-
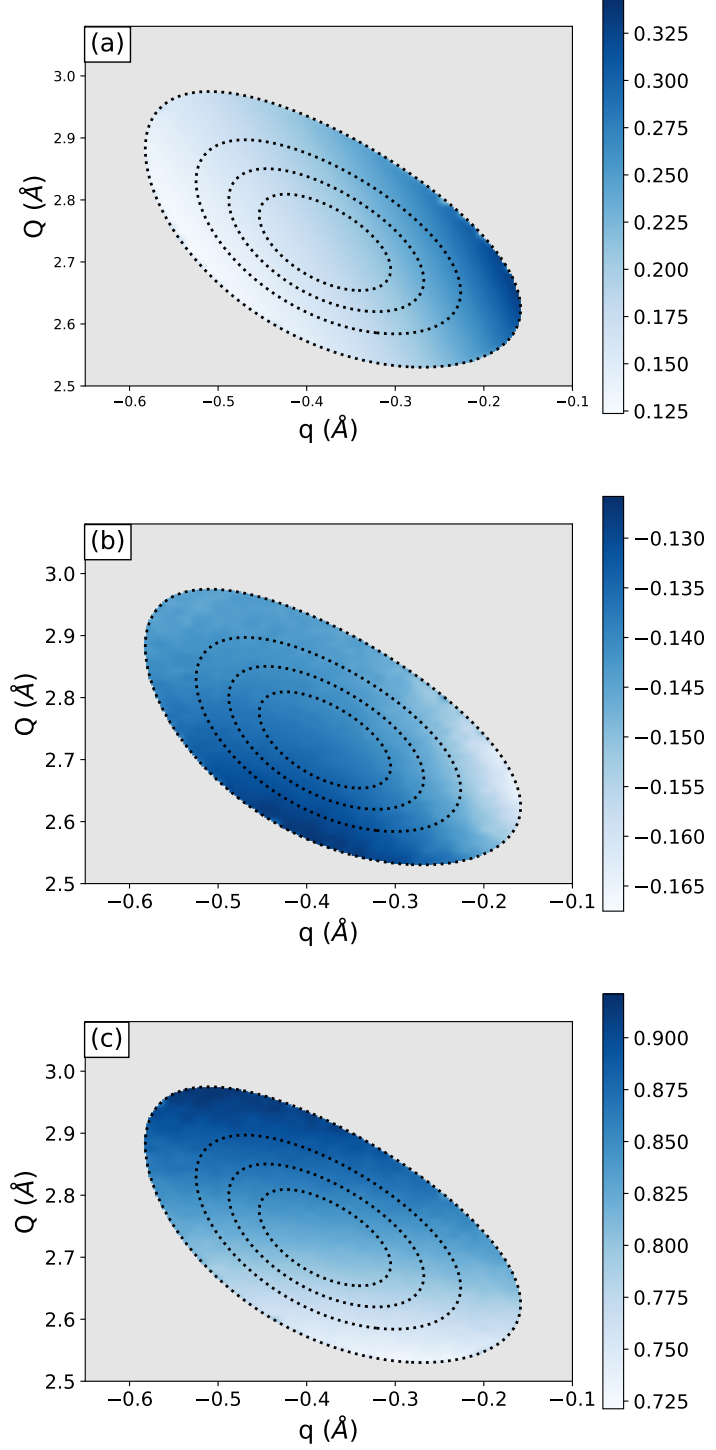

FIG. 6. Color map of the normalized matrix elements of $\kappa_{2}(q, Q)$ for the asymmetric hydrogen bond model at $300 \mathrm{~K}$. Panels (a) and (c) represent the diagonal elements corresponding respectively to the light and heavy degrees of freedom. Panel (b) shows the off-diagonal matrix element. The contour plots of the probability density are superimposed (dotted black curve). The gray area outside of the lowest probability contour is not statistically relevant and it was thus excluded.

tailed in appendix D. Here, we point out two important differences with the one-dimensional case. Firstly, the momentum distribution becomes a generalized Gaussian with a $N \times N$ position-dependent inverse covariance matrix $\kappa_{2}(\mathbf{q})$, where $N$ is the number of degrees of freedom of the physical system. Secondly, higher order terms in the Edgeworth expansion become tensors of the same order of the term in the series. For example, the evaluation of EW4 necessitates estimating $N^{4}$ entries. The growth 
of the number of entries, together with the increasing numerical cost of the estimate of each term, is a serious challenge for extending the method to many-dimensional systems. Note that, since each term can be estimated independently of the others, trivial parallelization of the code can be employed in view of challenging applications. These difficulties notwithstanding, the control on convergence available via the expansion is an important conceptual advantage compared to alternative methods.

Figure 5.a and 5.b show the position and momentum probability distributions, respectively, which are obtained by integration of the Wigner density:

$$
\begin{aligned}
& \mathbb{P}(q, Q)=\int \mathrm{d} p \mathrm{~d} P W(q, p, Q, P) \\
& \mathbb{P}(p, P)=\int \mathrm{d} q \mathrm{~d} Q W(q, p, Q, P)
\end{aligned}
$$

The figures show that, for these quantities, the Edgeworth expansion is already converged at order zero and the WiLD method almost perfectly reproduces the reference probability distributions. In this case, the WiLD simulation is able to capture a variety of nuclear quantum effects. First, we note that, at fixed $Q$ the quantum distribution in Fig. 5.a is much wider than the classical one because of the high zero-point energy of the light degree of freedom. Moreover, the zero-point motion is responsible for a large shift of the equilibrium position: quantum effects shorten the bond length and shift the Hydrogen mean position towards the center of the bond.

In addition, in this $2 \mathrm{D}$ system, the $p$ and $P$ momenta are correlated, as apparent in Fig. 5.b. The classical Boltzmann momentum distribution is separable and can be written as a product of non-correlated Gaussian factors. However, in quantum mechanics, this distribution is not necessarily Gaussian due to non-commutation of the position and momentum operators, and momenta associated with different degrees of freedom can be correlated. This is the case for the asymmetric hydrogen bond model, as can be seen from the tilt of the 2D momentum distribution in figure 5.b. The correlation in momentum space is indeed captured in the $2 \times 2$ matrix $\kappa_{2}(q, Q)$ and it is therefore perfectly accounted for by the WiLD simulations, even at the order zero of the Edgeworth expansion. Fig. 6 shows the matrix elements of $\kappa_{2}(q, Q)$ normalized by $\lambda_{q}^{2}$ and $\lambda_{Q}^{2}$ (for the diagonal elements in panels a and c, respectively) or by $\lambda_{q} \lambda_{Q}$ (for the off-diagonal element in panel b). In the classical limit, the diagonal elements would be constant and equal to one while the off-diagonal elements would be null. In contrast, in Fig. 6.a, the diagonal element for the light degree of freedom goes down as low as 0.125 , indicating large zero-point energy effects. It also displays a marked dependence on position, with almost a factor of 3 between its lowest and its highest value, revealing a high degree of non-classical positionmomentum correlation. As expected, the diagonal element for the heavy degree of freedom, Fig. 6.c, is closer to one but it still deviates from its classical value and varies with position (although more weakly). Finally, the off- diagonal element in Fig. 6.b is non-zero and causes the correlation in momentum space mentioned above.

\section{CONCLUSIONS}

In this paper, we introduced a new method to sample the quantum Wigner thermal density, conveniently expressed as an Edgworth expansion. In this form, the Wigner density is given by the product of a positive exponential function (EW0) times a series of even powers of the momentum that converges asymptotically to the exact result and can originate negative values. The new sampling method is based on a generalized Langevin equation that rigorously conserves the, usually dominant, EW0 part of the Wigner density. The equations of motion of the Wigner-Langevin dynamics (WiLD) display several non-standard features, namely the presence of a generalized force that couples momenta and coordinates and a position-dependent friction term. These quantities are defined as averages over an appropriate conditional probability density and are, in general, not known analytically. An algorithm to propagate the WiLD equations on the basis of accurate path-integral estimates of the forces and friction was presented and tested on a typical set of one and two-dimensional model systems. The algorithm proved as accurate as a recently proposed method based on generalized Monte Carlo sampling, its convergence can be systematically checked, and non-trivial quantum effects (such as zero point energy, momentum-position and momentum-momentum correlations) are reliably captured. We also showed, for the notoriously difficult case of the quartic potential at low temperature, that including higher order terms in the Edgeworth approximation enables to detect negative regions of the Wigner density and improves the estimate of the mean square momentum. More in general, these terms capture non-Gaussian features in the momentum distribution that can have measurable experimental effects, for example, in Compton scattering ${ }^{43}$.

In its current form, the WiLD is slightly more expensive than its Monte Carlo counterpart, by a factor that we estimate between two and five for the models explored here. The brute force implementation of the method presented here is problematic for systems with many degrees of freedom due to unfavourable scaling of the auxiliary calculations required for the cumulants and the generalised force. Future improvements, such as noise compensation, are likely to close the gap and allow for applications to high-dimensional systems. Furthermore, the auxiliary calculations can be trivially parallelized to enable time-effective implementations of the method. Interestingly, the work presented here paves the way to applications beyond the capability of the Monte Carlo scheme. In particular, since the sampling is performed in a dynamics, it is possible to employ the WiLD trajectories to compute not only static, but also time-dependent properties of the system. In fact, in analogy with the popular 
ring-polymer ${ }^{44-46}$ and centroid ${ }^{47,48}$ molecular dynamics approaches, the WiLD is exact in the classical and harmonic cases (at least in the small friction limit or if an appropriate scheme is used to deconvolute the effect of the thermostat ${ }^{49}$ ) and it conserves the positive part EW0 of the Wigner density. The results presented here demonstrate that WiLD is a reliable sampling dynamics for the Wigner function and therefore provides accurate static quantum averages or initial conditions for linearized calculations of time-correlation functions. Future work will explore its performance as a tool for computing dynamical quantities directly and compare its results with recent alternatives that aim at preserving approximations to the positive definite part of the Wigner density ${ }^{19-22}$.

\section{SUPPLEMENTARY MATERIAL}

The supplementary material details the derivation of the integration method for the auxiliary Langevin dynamics. It is an adaptation to the open chain of the Path-Integral Ornstein-Uhlenbeck Dynamics (PIOUD) of ref. 36 .

\section{ACKNOWLEDGEMENT}

The authors acknowledge the Germaine De Staël exchange program for providing mobility funds that greatly facilitated the completion of this work. Support from the European Union?s Horizon 2020 research and innovation program under the grant agreement No. 676531, project E-CAM, is also acknowledged.

\section{Appendix A: Derivation of the equations of motion}

\section{WiLD equations of motion}

In this section we derive the WiLD equations of motion and show that they sample the modified Boltzmann distribution $\mathcal{P}(q, p) \propto e^{-\beta H_{\text {eff }}(q, p)}$ (with $H_{\text {eff }}$ defined in eq. (10) of the main text). The logic of our proof is as follows. We postulate a form for the generalised Langevin equation containing unknown functions that we then determine by substituting the dynamical system, together with the modified Boltzmann distribution, in the FokkerPlanck equation and enforcing stationarity of the probability density. More in detail, let us consider the following generalized Langevin equations:

$$
\left\{\begin{array}{l}
\dot{q}=p / m \\
\dot{p}=\mathcal{F}(q, p)+\sigma(q) R(t)
\end{array}\right.
$$

where $\mathrm{R}(\mathrm{t})$ is a white noise, and $\mathcal{F}(q, p)$ is of the form,

$$
\mathcal{F}(q, p)=A(q)+B(q) p+C(q) p^{2}
$$

$A, B$ and $C$ are functions of the position that we specify below. The Fokker-Planck equation describes the timeevolution of the phase-space probability distribution $\mathcal{P}$ for the stochastic dynamics (A1):

$$
\frac{\partial \mathcal{P}}{\partial t}=-\frac{p}{m} \frac{\partial \mathcal{P}}{\partial q}-\frac{\partial}{\partial p}[\mathcal{F} \times \mathcal{P}]+\frac{\sigma^{2}}{2} \frac{\partial^{2} \mathcal{P}}{\partial p^{2}}
$$

Note that the evolution equation (A3) for the probability density could also be obtained from the combined use of the continuity equation and the Furutsu-Novikov theorem as in Ref. 50. We then use (A3) to determine the expressions of the functions $A, B$ and $C$ such that the probability density $\mathcal{P}(q, p) \propto e^{-\beta H_{\text {eff }}(q, p)}$ is stationary (i.e. $\frac{\partial \mathcal{P}}{\partial t}=0$ ). The Fokker-Planck equation yields (we omit the dependence on position to simplify the notations):

$$
\begin{aligned}
& \frac{\beta p}{m}\left(\frac{p^{2}}{2 m \lambda^{2}} \frac{\partial \kappa_{2}}{\partial q}+\frac{\partial U}{\partial q}\right)+\frac{\kappa_{2}}{\lambda^{2}} \frac{\beta p}{m}\left(A+B p+C p^{2}\right) \\
& -(B+2 C p)+\frac{\sigma^{2}}{2}\left(-\frac{\beta \kappa_{2}}{m \lambda^{2}}+\left(\frac{\beta \kappa_{2}}{m \lambda^{2}} p\right)^{2}\right)=0
\end{aligned}
$$

The equation above is satisfied by setting to zero the position-dependent coefficient for each power of $p$. This leads to the following expressions for the unknown functions in eq. (A2):

$$
\begin{aligned}
C(q) & =-\frac{1}{2 m \kappa_{2}(q)} \frac{\partial \kappa_{2}}{\partial q} \\
B(q) & =-\frac{\kappa_{2}(q)}{\lambda^{2}} \frac{\beta \sigma(q)^{2}}{2 m}=-\gamma(q) \\
A(q) & =-\frac{\lambda^{2}}{\kappa_{2}(q)} \frac{\partial U}{\partial q}-\frac{\lambda^{2}}{\kappa_{2}^{2}(q)} \beta^{-1} \frac{\partial \kappa_{2}}{\partial q}
\end{aligned}
$$

Inserting these expressions into (A1) leads to the WiLD equations of motion, eq. (11) of the main text, and the discussion above shows that the EW0 probability density $e^{-\beta H_{\text {eff }}(q, p)}$ is stationary under this stochastic dynamics. Equation (A6) is the fluctuation-dissipation relation of the WiLD. In the main text, we considered the case where $\sigma$ is constant and consequently $\gamma(q)$ is proportional to $\kappa_{2}(q)$, but the relation could also be enforced with a position-independent friction coefficient $\gamma$, if $\sigma(q)^{2} \propto 1 / \kappa_{2}(q)$. Furthermore, it is interesting to note that, with these equations of motion, the conserved quantity in the dynamics obtained by setting $\sigma=0$ in eq. (A8) is not simply the effective Hamiltonian $H_{\text {eff }}(q, p)$, but the $\operatorname{sum} H_{\text {eff }}(q, p)+\beta^{-1} \ln \frac{\kappa_{2}(q)}{\lambda^{2}}$.

As illustrated in the next subsection, the stochastic dynamics derived above is not the only available choice for sampling the modified Boltzmann distribution. In this work, we choose to explore the performance of this specific scheme in view of future calculations of timedependent statistical quantities. The WiLD dynamics, in fact, satisfies (see Appendix B) two conditions usually invoked when extending the use of dynamics that sample 
the quantum probability density to the evaluation of dynamical quantities: the correct classical limit is obtained and the dynamics is exact for the quantum harmonic oscillator.

\section{Alternative formulations}

Alternative equations of motion can be derived that also preserve the EW0 approximation to the Wigner density and might possibly be more convenient if using the stochastic dynamics with the only purpose of sampling the Wigner density. In the following, we briefly discuss two possible alternatives to the dynamical system (A1) and (A2).

Firstly, the density $\mathcal{P}(q, p) \propto e^{-\beta H_{\text {eff }}(q, p)}$ can be sampled using the standard Hamilton equations for the effective Hamiltonian $H_{\text {eff }}$ and adding the Langevin friction and random force, which yields the following equations of motion:

$$
\left\{\begin{array}{l}
\dot{q}=\frac{\kappa_{2}(q)}{\lambda^{2}} p / m \\
\dot{p}=-\frac{\partial U}{\partial q}-\frac{p^{2}}{2 m \lambda^{2}} \frac{\partial \kappa_{2}}{\partial q}-\gamma(q) p+\sigma(q) R(t)
\end{array}\right.
$$

where the coefficients $\gamma(q)$ and $\sigma(q)$ are related by the fluctuation-dissipation relation (A6). In this formulation, the force has a slightly simpler expression but the relation between momentum and velocity is non-trivial and depends on position. For practical purposes however, these alternative equations present the advantage that they no longer require computing the inverse of $\kappa_{2}(q)$ and that the force term does not involve products of noisy estimates as in the WiLD case.

A second alternative approach could be used to sample the EW0 distribution, in which the equations of motion are obtained through change of variable in momentum space. Let us write the density $\mathcal{P}(p, q)$ as:

$\mathcal{P}(p, q)=\frac{1}{\mathcal{N}} \int \mathrm{d} p^{\prime} \delta\left(p-p^{\prime}\right) \exp \left\{-\beta\left(\frac{\kappa_{2}(q)}{\lambda^{2}} \frac{p^{\prime 2}}{2 m}-U(q)\right)\right\}$

where $\mathcal{N}$ is a normalization constant. We then perform the change of variable $p^{\prime}=\sqrt{\frac{\lambda^{2}}{\kappa_{2}(q)}} \tilde{p}$ and obtain:

$$
\begin{aligned}
\mathcal{P}(p, q)=\frac{1}{N} \int \mathrm{d} \tilde{p} & \left|\frac{\lambda^{2}}{\kappa_{2}(q)}\right|^{1 / 2} \delta\left(p-\sqrt{\lambda^{2} / \kappa_{2}(q)} \tilde{p}\right) \\
& \times \exp \left\{-\beta\left(\frac{\tilde{p}^{2}}{2 m}-U(q)\right)\right\} \quad
\end{aligned}
$$

The exponential factor in this expression is now a simple Boltzmann distribution with the (modified) potential $U(q)$, and it can be sampled in the phase space $(q, \tilde{p})$ using a standard Langevin dynamics at inverse temperature $\beta$ :

$$
\left\{\begin{array}{l}
\dot{q}=\frac{\tilde{p}}{m} \\
\dot{\tilde{p}}=-\frac{\partial U}{\partial q}-\gamma \tilde{p}+\sigma R(t) \text { with } \gamma=\frac{\beta \sigma^{2}}{2 m}
\end{array}\right.
$$

To compute mean values of observables or histograms of the EW0 distribution, one should then transform back the sampling momentum $\tilde{p}$ to the physical momentum $p$ and reweight the estimates using the factor $\left|\frac{\lambda^{2}}{\kappa_{2}(q)}\right|^{1 / 2}$. This alternative formulation presents the advantage that it does not require computing the derivative $\frac{\partial \kappa_{2}}{\partial q}$, since the conservative part of the dynamics only involves the simple force term $\frac{\partial U}{\partial q}$, while quantum effects such as position-momentum and momentum-momentum correlations are recovered a posteriori through the positiondependent change of variable in momentum space.

These two alternative formulations might be interesting to consider for the purposes of optimizing the EW0 sampling efficiency. However, in contrast to the WiLD equations of motion presented in the main text and in appendix A 1, the trajectories generated within these alternative schemes cannot be used for direct calculation of dynamical properties. In particular, in the harmonic case, the vibration frequencies obtained with equations (A8) or (A11) are modified with respect to the actual physical frequencies, hindering their use to compute dynamical properties directly. On the contrary, the WiLD equations of motion preserve the natural (classical) interpretation of momenta and we show in appendix B that they provide valid dynamical results in both the harmonic and classical limits.

\section{Appendix B: Classical and harmonic limits of the dynamics}

In this section, we discuss the classical and harmonic limits of the WiLD equations of motion (A8) (or eq. (11) of the main text). We show that the force $\mathcal{F}(q, p)$ reduces to its classical expression and that the Edgeworth factor $C_{E W}(q, p)$ tends to 1 so that, in both cases, the dynamical properties obtained in WiLD simulations are correct (at least, as always in Langevin propagation, in the limit $\sigma \rightarrow 0)$.

To simplify the notations, we define:

$$
f(q, \Delta) \equiv\left\langle q-\frac{\Delta}{2}\left|e^{-\beta \hat{H}}\right| q+\frac{\Delta}{2}\right\rangle
$$

and rewrite the auxiliary sampling density $\rho_{c}$ as:

$$
\rho_{c}(\Delta \mid q)=\frac{f(q, \Delta)}{\int d \Delta f(q, \Delta)}
$$

\section{Classical limit}

In the classical, high temperature, limit, the Trotter break-up of the kinetic and potential operators in the 
quantum Hamiltonian can be applied directly to obtain:

$$
\begin{aligned}
e^{-\beta \hat{H}} & =e^{-\frac{m \lambda^{2}}{\hbar^{2}} \hat{H}} \\
& =e^{-\frac{m \lambda^{2}}{2 \hbar^{2}} V(\hat{q})} e^{-\frac{\lambda^{2} \hat{p}^{2}}{2 \hbar^{2}}} e^{-\frac{m \lambda^{2}}{2 \hbar^{2}} V(\hat{q})}+\mathcal{O}\left(\lambda^{6}\right)
\end{aligned}
$$

In the equation above, we have used the relation $\beta=$ $m \lambda^{2} / \hbar^{2}$ to explicitate the role of the thermal De Broglie wavelength, a more suitable control parameter than the temperature for the classical limit (see below). Eq. (B1) then becomes:

$$
\begin{aligned}
f(q, \Delta) \propto \exp \left\{-\frac{m \lambda^{2}}{2 \hbar^{2}}(V(q\right. & \left.\left.\left.-\frac{\Delta}{2}\right)+V\left(q+\frac{\Delta}{2}\right)\right)\right\} \\
\times & \exp \left\{-\frac{\Delta^{2}}{2 \lambda^{2}}\right\}
\end{aligned}
$$

Due to the Gaussian factor in this expression, only values of $\Delta$ at most of the order of the De Broglie wavelength will lead to significant contributions to the Wigner density. Let us now consider the different terms in the generalized force and friction coefficients in eqns (12) and (13) of the main text. We begin by performing the change of variable $X=\Delta / \lambda$ to obtain:

$$
\begin{aligned}
\kappa_{2}(q) & =\frac{\int \mathrm{d} X \lambda^{2} X^{2} f(q, \lambda X)}{\int \mathrm{d} X f(q, \lambda X)} \\
\frac{\partial U}{\partial q} & =\frac{\int \mathrm{d} X \frac{1}{2}\left[V^{(1)}\left(q-\frac{\lambda X}{2}\right)+V^{(1)}\left(q+\frac{\lambda X}{2}\right)\right] f(q, \lambda X)}{\int \mathrm{d} X f(q, \lambda X)}
\end{aligned}
$$

where $V^{(1)}$ is the first derivative of the physical potential $V$. The expressions above are integrals over the density $f(q, \lambda X) / \int \mathrm{d} X f(q, \lambda X)$ that we can rewrite via a Taylor expansion in powers of $\lambda$ :

$$
\begin{aligned}
\frac{f(q, \lambda X)}{\int \mathrm{d} X f(q, \lambda X)}= & \left(1+\frac{m \lambda^{4}}{8 \hbar^{2}} V^{(2)}(q)\left(1-X^{2}\right)+\mathcal{O}\left(\lambda^{6}\right)\right) \\
& \times \frac{e^{-X^{2} / 2}}{\int \mathrm{d} X e^{-X^{2} / 2}}
\end{aligned}
$$

where $V^{(n)}$ denotes $n$-th order derivative of the physical potential $V$. Substituting (B6) in eq. (B5) and performing the integrals, we obtain:

$$
\begin{aligned}
\kappa_{2}(q) & =\lambda^{2}+\mathcal{O}\left(\lambda^{6}\right) \\
\frac{\partial U}{\partial q} & =V^{(1)}(q)+\frac{\lambda^{2}}{8} V^{(3)}(q)+\frac{\lambda^{4}}{128} V^{(5)}(q)+\mathcal{O}\left(\lambda^{6}\right)
\end{aligned}
$$

Note that the (quantum) definition of $U(q)$, eq. (5) of the main text, has some analogies with the definition of a quantum free energy at position $q$. Its derivative can then be interpreted as a potential of mean force incorporating non-local contributions via the integral over the displacement $\Delta$. Eq. (B7) shows that in the classical limit, this mean force reduces to $-V^{(1)}(q)$, the standard force. Higher order terms in the $\lambda$ expansion denote the quantum delocalization by implicitly exploring broader regions of the potential via higher order derivatives.

To complete the estimate of the terms in eq. (12), we observe that $p$ is distributed according to $e^{-\frac{\kappa_{2} p^{2}}{2 \hbar^{2}}}$, so that we can assign it the order of magnitude $p^{2} \sim \hbar^{2} / \lambda^{2}$. This, together with (B7), yields:

$$
\begin{aligned}
F(q, p)= & -V^{(1)}(q)-\frac{\lambda^{2}}{8} V^{(3)}(q) \\
& +\left(\frac{\hbar^{2}}{2 m \lambda^{2}}+\frac{p^{2}}{2 m}\right) \frac{m \lambda^{4}}{4 \hbar^{2}} V^{(3)}(q)+\mathcal{O}\left(\lambda^{4}\right) \\
\sim & -V^{(1)}(q)+\mathcal{O}\left(\lambda^{2}\right)
\end{aligned}
$$

Similarly,

$$
\gamma(q)=\frac{\kappa_{2}(q)}{\lambda^{2}} \frac{\beta \sigma^{2}}{2 m} \sim \frac{\beta \sigma^{2}}{2 m}+\mathcal{O}\left(\lambda^{6}\right)
$$

In summary, in the high-temperature limit $\lambda \rightarrow 0$, the WiLD equations of motion (11) become:

$$
\left\{\begin{array}{l}
\dot{q}=p / m \\
\dot{p}=-V^{(1)}(q)-\frac{\beta \sigma^{2}}{2 m} p+\sigma R(t)
\end{array}\right.
$$

Thus, we retrieve the standard classical Langevin equation for a system subject to potential $V$ at inverse temperature $\beta$. The Edgeworth expansion terms can be estimated with the same approach. In particular, we find that $\kappa_{4}(q)=\mathcal{O}\left(\lambda^{6}\right)$ and, using $p^{4} \sim \hbar^{4} / \lambda^{4}, C_{E W}(q, p)=$ $1+\mathcal{O}\left(\lambda^{2}\right)$. In the classical limit $\lambda \rightarrow 0$, the Wigner density approaches the classical Boltzmann density, which is a Gaussian function of $p$, therefore the Edgeworth expansion tends to converge already at order EW0 in that limit. Indeed, as we showed for the 4 th order, even though $p^{n}$ tends to take large values in the high-temperature limit, the high-order cumulants $\kappa_{n}$ should vanish even faster, so that the EWn terms tend to zero for $\lambda \rightarrow 0$.

\section{Harmonic case}

We now consider the case of the harmonic potential $V(q)=\frac{m \omega^{2}}{2} q^{2}$. For this system, the density matrix elements in the coordinate representation are known analytically (see for example ref. 51), and we have:

$$
\begin{aligned}
f(q, \Delta) \propto \exp \left\{-\frac{m \omega}{4 \hbar} \tanh ^{-1}(\beta \hbar \omega / 2) \Delta^{2}\right\} \\
\times \exp \left\{-\frac{m \omega}{\hbar} \tanh (\beta \hbar \omega / 2) q^{2}\right\}
\end{aligned}
$$

so that

$$
\begin{aligned}
\kappa_{2}(q) & =\frac{\int \mathrm{d} \Delta \Delta^{2} \exp \left\{-\frac{m \omega}{4 \hbar} \tanh ^{-1}(\beta \hbar \omega / 2) \Delta^{2}\right\}}{\int \mathrm{d} \Delta \exp \left\{-\frac{m \omega}{4 \hbar} \tanh ^{-1}(\beta \hbar \omega / 2) \Delta^{2}\right\}} \\
& =\frac{2 \hbar}{m \omega} \tanh (\beta \hbar \omega / 2)
\end{aligned}
$$


Note that $\kappa_{2}$ is independent of $q$ and $\frac{\partial \kappa_{2}}{\partial q}=0$. We can also write an analytic expression for $\frac{\partial U}{\partial q}$ via:

$$
\begin{aligned}
\frac{\partial U}{\partial q} & =-\frac{1}{\beta} \frac{\int \mathrm{d} \Delta \frac{\partial f}{\partial q}(q, \Delta)}{\int \mathrm{d} \Delta f(q, \Delta)} \\
& =\frac{2 m \omega}{\beta \hbar} q \tanh (\beta \hbar \omega / 2)
\end{aligned}
$$

Thus, for a harmonic system, the WiLD equations of motion are given by:

$$
\left\{\begin{array}{l}
\dot{q}=p / m \\
\dot{p}=-m \omega^{2} q-\frac{\sigma^{2}}{2 m \Theta(\beta, \omega)} p+\sigma R(t)
\end{array}\right.
$$

where $\Theta(\beta, \omega)=\frac{\hbar \omega}{2} \operatorname{coth}(\beta \hbar \omega / 2)$ is the effective quantum thermal energy of the harmonic oscillator. Therefore, the Wigner-Langevin dynamics reduces to the classical evolution for its the deterministic part (i.e. $F(q, p)$ in eq. (11) is simply the classical force), combined with a Langevin thermostat at an effective temperature $\Theta(\beta, \omega) / k_{B}$.

Finally, note that, in the harmonic oscillator case, since $f(q, \Delta)$ is Gaussian in $\Delta$, all cumulants of order higher than two are zero and $C_{E W}(q, p)=1$.

\section{Appendix C: Auxiliary path integral sampling}

As discussed in sec. III A, at each time step of the main Wigner-Langevin dynamics, an auxiliary path integral calculation is performed to compute the non-analytical force and the friction coefficient appearing in the generalized equations of motion. These quantities depend on the functions $\kappa_{2}(q), \frac{\partial U}{\partial q}$ and $\frac{\partial \kappa_{2}}{\partial q}$, which are expressed as averages over the path integral density $\rho_{\nu}$. In this appendix, we first obtain the expression of the path integral estimators in equations (16), (17) and (18) of the main text, then we derive a set of Langevin equations to sample the density $\rho_{\nu}$, and finally, we summarize the numerical integration scheme used for this auxiliary Langevin calculation. Further details on this algorithm can be found in the supplementary material.

Let us start by recalling the expression of $\rho_{\nu}$ (equation (15) of the main text):

$$
\begin{aligned}
& \rho_{\nu}\left(\Delta, x_{1}, \ldots, x_{\nu-1} \mid q\right) \propto \\
& \exp \left\{-\frac{\beta}{\nu}\left[\frac{1}{2} V\left(q+\frac{\Delta}{2}\right)+\frac{1}{2} V\left(q-\frac{\Delta}{2}\right)+\sum_{\lambda=1}^{\nu-1} V\left(x_{\lambda}\right)\right]\right\} \\
& \times \exp \left\{-\frac{m \nu}{2 \beta \hbar^{2}}\left[\left(q-\frac{\Delta}{2}-x_{\nu-1}\right)^{2}+\left(q+\frac{\Delta}{2}-x_{1}\right)^{2}\right]\right\} \\
& \times \exp \left\{-\frac{m \nu}{2 \beta \hbar^{2}} \sum_{\lambda=1}^{\nu-2}\left(x_{\lambda+1}-x_{\lambda}\right)^{2}\right\}
\end{aligned}
$$

In this equation, $\nu$ is the number of path-integral slices and $x_{1}, \ldots, x_{\nu-1}$ are the positions of the path-integral beads. Since we consider off-diagonal density matrix elements, their path integral representation corresponds to an open chain of harmonically coupled beads stretching between $q-\frac{\Delta}{2}$ and $q+\frac{\Delta}{2}$ (see the inset of figure 1). Note that the probability density $\rho_{\nu}$ is conditional to $q$, the current position of the physical degree of freedom in the WiLD trajectory. In the auxiliary path integral sampling described in the following of this appendix, the value of $q$ is thus fixed and the endpoints, $q-\frac{\Delta}{2}$ and $q+\frac{\Delta}{2}$, are allowed to fluctuate around it.

\section{Path integral estimators}

In this first subsection, we derive equations (16), (17) and (18) of section II C.

Equation (16) follows directly from the definition of $\kappa_{2}(q)$ in equation (7). To obtain equation (17), let us first rewrite equation (5) as:

$$
\begin{aligned}
U(q) & =-\frac{1}{\beta} \ln \int \mathrm{d} \Delta f(q, \Delta) \\
\Rightarrow \frac{\partial U}{\partial q} & =-\frac{\int \mathrm{d} \Delta \frac{\partial f}{\partial q}}{\beta \int \mathrm{d} \Delta f(q, \Delta)}
\end{aligned}
$$

Note that from the path-integral expression (C1), it follows that $f(q, \Delta)$ is strictly positive so that the logarithm in equation $(\mathrm{C} 2)$ is well defined. Using equation (C1) to express $f$ and performing the change of variables $y_{\lambda}=x_{\lambda}-q$ for $\lambda=1, \ldots, \nu-1$, one can write:

$$
\begin{array}{r}
\frac{\partial U}{\partial q}=\int \mathrm{d} \Delta \mathrm{d} y_{1} \ldots \mathrm{d} y_{\nu-1} \rho_{\nu}\left(\Delta, y_{1}+q, \ldots, y_{\nu-1}+q \mid q\right) \\
\times \frac{1}{\nu}\left[\frac{1}{2} \frac{\partial}{\partial q} V\left(q+\frac{\Delta}{2}\right)+\frac{1}{2} \frac{\partial}{\partial q} V\left(q-\frac{\Delta}{2}\right)\right. \\
\left.+\sum_{\lambda=1}^{\nu-1} \frac{\partial}{\partial q} V\left(q+y_{\lambda}\right)\right]
\end{array}
$$

To proceed, note that $\frac{\partial}{\partial q} V\left(q+y_{\lambda}\right)=\frac{\partial}{\partial y_{\lambda}} V\left(q+y_{\lambda}\right)$ and, since $d y_{\lambda}=d x_{\lambda}$, going back to the original variables $x_{\lambda}$ yields equation (17).

Finally we derive equation (18) : from the definition of $\kappa_{2}(q)$ we can write

$$
\begin{aligned}
\frac{\partial \kappa_{2}}{\partial q}= & \int \mathrm{d} \Delta \Delta^{2} \frac{\partial}{\partial q} \rho_{c}(\Delta \mid q) \\
= & \int \mathrm{d} \Delta \Delta^{2} \frac{\frac{\partial f}{\partial q}}{\int \mathrm{d} \Delta f(q, \Delta)} \\
& -\frac{\int \mathrm{d} \Delta \frac{\partial f}{\partial q}}{\int \mathrm{d} \Delta f(q, \Delta)} \int \mathrm{d} \Delta \Delta^{2} \rho_{c}(\Delta \mid q)
\end{aligned}
$$

In the last line, we recognize the product of $\frac{\partial U}{\partial q}$ and $\kappa_{2}(q)$. The same change of variable as used for eq. (C3) leads to the path integral estimator in equation (18). 


\section{Avoiding systematic bias}

As indicated in Sec. III B, it is important to avoid systematic errors induced by correlated noise in the estimators discussed in Appendix C 1 . The evaluation of $1 / \kappa_{2}$, see eq. (12), might also introduce a systematic bias to the force. This can be prevented using a correction term proportional to the variance on the estimation of $\kappa_{2}$. To show it, let us introduce the following notation:

$$
\tilde{\kappa}_{2}=\kappa_{2}+X
$$

The symbol $\tilde{\kappa}_{2}$ denotes the estimated value obtained from the auxiliary sampling. If $\tilde{\kappa}_{2}$ is an unbiased estimator, $X$ is a random variable with zero mean. We then use the expansion:

$$
\frac{1}{\tilde{\kappa}_{2}}=\frac{1}{\kappa_{2}+X} \approx \frac{1}{\kappa_{2}}-\frac{X}{\kappa_{2}^{2}}+\frac{X^{2}}{\kappa_{2}^{3}}
$$

to obtain the following unbiased estimator for $1 / \kappa_{2}$ :

$$
\frac{1}{\kappa_{2}} \approx \frac{1}{\tilde{\kappa}_{2}}-\frac{\sigma_{\tilde{\kappa}_{2}}^{2}}{\tilde{\kappa}_{2}^{3}}
$$

where $\sigma_{\tilde{\kappa}_{2}}^{2}$ is the variance of the random variable $\tilde{\kappa}_{2}$. This variance is estimated numerically using the $N_{\text {traj }}$ uncorrelated auxiliary Langevin dynamics performed at each time step. Similarly, the unbiased estimator for $1 / \kappa_{2}^{2}$ is:

$$
\frac{1}{\kappa_{2}^{2}} \approx \frac{1}{\tilde{\kappa}_{2}^{2}}-3 \frac{\sigma_{\tilde{\kappa}_{2}}^{2}}{\tilde{\kappa}_{2}^{4}}
$$

A generalization of this correction to the multidimensional case is provided in appendix D.

\section{Langevin sampling of $\rho_{\nu}$}

$\rho_{\nu}$ is sampled via a classical Langevin dynamics. To derive the appropriate Langevin equations, one should first attach fictitious momenta to the path-integral degrees of freedom. We thus sample the density:

$$
\begin{aligned}
\rho_{\mathrm{lgv}} \propto & \rho_{\nu}\left(\Delta, x_{1}, \ldots, x_{\nu-1} \mid q\right) \\
& \times \exp \left\{-\frac{\beta}{\nu}\left[\frac{p_{\Delta}^{2}}{2 m_{\Delta}}+\sum_{\lambda=1}^{\nu-1} \frac{p_{\lambda}^{2}}{2 m_{\lambda}}\right]\right\}
\end{aligned}
$$

with $p_{\Delta}$ the momentum associated to $\Delta$ with fictitious mass $m_{\Delta}$ and $p_{\lambda}$ the momenta associated to $x_{\lambda}$ with fictitious mass $m_{\lambda}(\lambda=1, \ldots, \nu-1)$. Standard Langevin equations are then derived for the open chain:

$$
\left\{\begin{aligned}
\dot{x}_{\lambda}= & p_{\lambda} / m_{\lambda} \\
\dot{p}_{\lambda}= & -m \omega_{0}^{2}\left(2 x_{\lambda}-x_{\lambda+1}-x_{\lambda-1}\right)-\frac{\partial}{\partial x_{\lambda}} V\left(x_{\lambda}\right) \\
& -\gamma_{\lambda} p_{\lambda}+\sigma_{\lambda} R_{\lambda}(t) \\
\dot{\Delta}= & p_{\Delta} / m_{\Delta} \\
\dot{p}_{\Delta}= & -\frac{1}{2} m \omega_{0}^{2}\left(\Delta-x_{1}+x_{\nu-1}\right) \\
& -\frac{1}{4}\left[\frac{\partial}{\partial q} V(q+\Delta / 2)-\frac{\partial}{\partial q} V(q-\Delta / 2)\right] \\
& -\gamma_{\Delta} p_{\Delta}+\sigma_{\Delta} R_{\Delta}(t)
\end{aligned}\right.
$$

with $\omega_{0}=\nu / \beta \hbar$ and where we have implicitly introduced the notation $x_{0}=q+\Delta / 2, x_{\nu}=q-\Delta / 2$. For every $\lambda$, the fluctuation-dissipation relation $\gamma_{\lambda}=\beta \sigma_{\lambda}^{2} / 2 m_{\lambda} \nu$ holds, and the $R_{\lambda}(t)$ are independent Gaussian white noises.

We used the Path-Integrals Ornstein-Uhlenbeck Dynamics (PIOUD) algorithm of Ref. 36 for the numerical integration of (C10). The method was originally derived for closed-chain path integral dynamics and we adapted it to the case of an open chain of harmonically coupled beads, as detailed in supplementary information. The PIOUD is a symmetric time-splitting scheme (as the one used for the WiLD integration) in which the harmonic coupling between the beads, the friction force and the random Langevin force are all integrated exactly in a single step, performed in the open chain normal mode basis. Different friction coefficients are also attached to each normal mode, with values chosen to optimize the sampling efficiency. Empirical observation shows that, with this integration procedure, the time step of the auxiliary dynamics does not necessarily need to be small compared to the period of the high-frequency internal vibrations of the open chain ${ }^{36}$ and can be chosen based on the relevant timescale associated with the physical potential $V$. Other time-splitting algorithms used in the context of closed-chain path integrals might also be adapted to the open chain problem, for example the BAOAB-num method introduced in Ref. 23, which yields the exact position distribution in the harmonic oscillator case, even for finite-size time step.

\section{Appendix D: Generalization to $N$ degrees of freedom}

The generalization of the WiLD formalism to $N$ degrees of freedom is non-trivial. In that case, $\kappa_{2}$ becomes a $N \times N$ matrix and care is required when deriving the Edgeworth expansion and the expressions for the generalized forces. 


\section{Edgeworth expansion}

Introducing the notation $\mathbf{q}, \mathbf{p}$ and $\boldsymbol{\Delta}$ to indicate $N$ dimensional vectors and taking into account the fact that $\kappa_{2}$ is now a matrix, equation (4) becomes:

$$
\begin{aligned}
& W(\mathbf{q}, \mathbf{p})=\frac{1}{(2 \pi \hbar)^{N} Q} \exp \left\{-\frac{\mathbf{p}^{\boldsymbol{\top}} \kappa_{2}(\mathbf{q}) \mathbf{p}}{2 \hbar^{2}}-\beta U(\mathbf{q})\right\} \\
& \times \int \mathrm{d} \boldsymbol{\Delta} \exp \left\{\left[\frac{1}{2 \hbar^{2}} \mathbf{p}^{\boldsymbol{\top}} \kappa_{2}(\mathbf{q})+\frac{i}{\hbar} \boldsymbol{\Delta}\right] \cdot \mathbf{p}\right\} \rho_{c}(\boldsymbol{\Delta} \mid \mathbf{q})
\end{aligned}
$$

where $U(\mathbf{q})$ and $\rho_{c}(\boldsymbol{\Delta} \mid \mathbf{q})$ are defined in analogy with eqs. (5) and (6). Introducing the notation:

$$
h(\mathbf{p})=\exp \left\{\left[\frac{1}{2 \hbar^{2}} \mathbf{p}^{\top} \kappa_{2}(\mathbf{q})+\frac{i}{\hbar} \boldsymbol{\Delta}\right] \cdot \mathbf{p}\right\}
$$

and considering the expansion of the exponential in $h$ gives:

$$
\begin{aligned}
h(\mathbf{p})=1 & +\sum_{i} p_{i} \frac{\partial h}{\partial p_{i}}(0)+\frac{1}{2 !} \sum_{i, j} p_{i} p_{j} \frac{\partial^{2} h}{\partial p_{i} \partial p_{j}}(0) \\
& +\frac{1}{3 !} \sum_{i, j, k} p_{i} p_{j} p_{k} \frac{\partial^{3} h}{\partial p_{i} \partial p_{j} \partial p_{k}}(0) \\
& +\frac{1}{4 !} \sum_{i, j, k, l} p_{i} p_{j} p_{k} p_{l} \frac{\partial^{4} h}{\partial p_{i} \partial p_{j} \partial p_{k} \partial p_{l}}(0)+\cdots
\end{aligned}
$$

where all the sum indices run from 1 to $N$.

Explicit calculation of the derivatives shows that odd orders in the expansion above contain only odd powers of the components of $\boldsymbol{\Delta}$ and, as in the 1D case, they vanish when integrated, by parity of $\rho_{c}$. Similarly to the $1 D$ case, we choose:

$$
\left[\kappa_{2}(\mathbf{q})\right]_{i j}=\int \mathrm{d} \boldsymbol{\Delta} \Delta_{i} \Delta_{j} \rho_{c}(\boldsymbol{\Delta} \mid \mathbf{q})
$$

With this choice of $\kappa_{2}$, the second order in the Taylor expansion cancels and we obtain:

$$
\begin{aligned}
& W(\mathbf{q}, \mathbf{p})=\frac{1}{(2 \pi \hbar)^{N} Q} \exp \left\{-\frac{\mathbf{p}^{\top} \kappa_{2}(q) \mathbf{p}}{2 \hbar^{2}}-\beta U(\mathbf{q})\right\} \\
& \times\left(1+\sum_{i, j, k, l} \frac{p_{i} p_{j} p_{k} p_{l}}{24 \hbar^{4}}\left[\left[\kappa_{4}(\mathbf{q})\right]_{i j k l}-\left[\kappa_{2}(\mathbf{q})\right]_{i j}\left[\kappa_{2}(\mathbf{q})\right]_{k l}\right.\right. \\
& \left.\left.-\left[\kappa_{2}(\mathbf{q})\right]_{i k}\left[\kappa_{2}(\mathbf{q})\right]_{j l}-\left[\kappa_{2}(\mathbf{q})\right]_{i l}\left[\kappa_{2}(\mathbf{q})\right]_{j k}\right]+\cdots\right)
\end{aligned}
$$

where $\kappa_{4}$ is a fourth order tensor defined by:

$$
\left[\kappa_{4}(\mathbf{q})\right]_{i j k l}=\int \mathrm{d} \boldsymbol{\Delta} \Delta_{i} \Delta_{j} \Delta_{k} \Delta_{l} \rho_{c}(\boldsymbol{\Delta} \mid \mathbf{q})
$$

\section{Langevin equations}

The multidimensional WiLD equations of motion that sample the probability density:

$$
\mathcal{P}(\mathbf{q}, \mathbf{p}) \propto \exp \left\{-\frac{\mathbf{p}^{\boldsymbol{\top}} \kappa_{2}(\mathbf{q}) \mathbf{p}}{2 \hbar^{2}}-\beta U(\mathbf{q})\right\}
$$

are determined similarly to the one-dimensional case in Appendix A 1. They read:

$$
\left\{\begin{array}{l}
\dot{\mathbf{q}}=M^{-1} \mathbf{p} \\
\dot{\mathbf{p}}=\mathbf{F}(\mathbf{q}, \mathbf{p})-\frac{\sigma^{2}}{2 \hbar^{2}} \kappa_{2}(\mathbf{q}) \mathbf{p}+\sigma \mathbf{R}(t)
\end{array}\right.
$$

where $M$ is the mass matrix of the system, $\sigma$ is a diagonal $N \times N$ matrix and $\mathbf{R}(t)$ is a vector of $N$ independent gaussian white noises. The force vector $\mathbf{F}$ is given by:

$$
\begin{aligned}
\mathbf{F}(\mathbf{q}, \mathbf{p})= & -\beta \hbar^{2} \kappa_{2}^{-1}(\mathbf{q}) M^{-1} \nabla \mathbf{U}(\mathbf{q}) \\
& +2 \hbar^{2} \kappa_{2}^{-1}(\mathbf{q}) \mathcal{V}(\mathbf{q})+\left(\begin{array}{c}
\mathbf{p}^{\top} C_{1}(\mathbf{q}) \mathbf{p} \\
\vdots \\
\mathbf{p}^{\top} C_{N}(\mathbf{q}) \mathbf{p}
\end{array}\right)
\end{aligned}
$$

We use the multidimensional Fokker-Planck equation to determine the expressions for the $N \times N$ matrices $C_{i}(\mathbf{q})$ $(i=1 \ldots N)$ and the $N$-dimensional vector $\mathcal{V}(\mathbf{q})$ in order for the probability density (D6) to be stationary:

$$
\begin{aligned}
& C_{i}(\mathbf{q})=-\frac{1}{2} \sum_{j=1}^{N} \frac{1}{m_{j}}\left[\kappa_{2}^{-1}(\mathbf{q})\right]_{i j} \frac{\partial \kappa_{2}}{\partial q_{j}} \\
& \mathcal{V}_{k}(\mathbf{q})=\sum_{i=1}^{N}\left[C_{i}(\mathbf{q})\right]_{i k}
\end{aligned}
$$

for $i$ and $k=1, \ldots, N$.

As in the 1-dimensional case, it is important to avoid systematic biases in the estimation of the generalized force. Following the idea illustrated in the main text, we make use of $N_{\text {traj }}$ independent estimated values for each nonanalytical term. Products of such terms are computed as averages over all possible combinations of uncorrelated estimators. For $\kappa_{2}^{-1}(q)$ we use the estimator:

$$
\kappa_{2}(q)^{-1} \simeq \tilde{\kappa}_{2}(q)^{-1}-\left\langle\tilde{\kappa}_{2}(q)^{-1} X \tilde{\kappa}_{2}(q)^{-1} X \tilde{\kappa}_{2}(q)^{-1}\right\rangle
$$

which corrects for the bias up to second order in the norm of $\kappa_{2}(q)^{-1} X$. In the equation above, we used the same notations as in appendix C 2: $\tilde{\kappa}_{2}(q)=\kappa_{2}(q)+X$ where $X$ is a random $N \times N$ matrix with zero mean value. Using the same approach described in appendix C 2, unbiased estimators are obtained also for the other terms appearing in the generalized force (D8), in particular those involving $C_{i}(\mathbf{q})$ and $\mathcal{V}(\mathbf{q})$.

In addition, due to the more complex form of the generalized force, the integration scheme described in section III A is slightly modified: steps $B_{1}$ and $B_{2}$ are combined and propagated using second order Runge Kutta algorithm. This maintains the accuracy of the overall algorithm up to order $\delta t^{2}$.

${ }^{1}$ R. P. Feynman, A. R. Hibbs, and D. F. Styer, Quantum mechanics and path integrals (Courier Corporation, 2010).

${ }^{2}$ D. Chandler and P. G. Wolynes, J. Chem. Phys. 74, 4078 (1981).

${ }^{3}$ D. M. Ceperley, Rev. Mod. Phys. 67, 279 (1995).

${ }^{4}$ M. Parrinello and A. Rahman, J. Chem. Phys. 80, 860 (1984). 
${ }^{5}$ M. Ceriotti, G. Bussi, and M. Parrinello, Phys. Rev. Lett. 103 030603 (2009).

${ }^{6}$ H. Dammak, Y. Chalopin, M. Laroche, M. Hayoun, and J.-J. Greffet, Phys. Rev. Lett. 103, 190601 (2009).

${ }^{7}$ H. Dammak, E. Antoshchenkova, M. Hayoun, and F. Finocchi, J. Phys. Condens. Matter 24, 435402 (2012).

${ }^{8}$ Y. Bronstein, P. Depondt, F. Finocchi, and A. M. Saitta, Phys. Rev. B 89, 214101 (2014).

${ }^{9}$ Y. Bronstein, P. Depondt, L. E. Bove, R. Gaal, A. M. Saitta, and F. Finocchi, Phys. Rev. B 93, 024104 (2016).

${ }^{10}$ M. Ceriotti, G. Miceli, A. Pietropaolo, D. Colognesi, A. Nale, M. Catti, M. Bernasconi, and M. Parrinello, Phys. Rev. B 82, 174306 (2010).

${ }^{11}$ E. Wigner, Phys. Rev. 40, 749 (1932).

${ }^{12}$ X. Sun, H. Wang, and W. H. Miller, J. Chem. Phys. 109, 7064 (1998).

${ }^{13}$ H. Wang, X. Sun, and W. H. Miller, J. Chem. Phys. 108, 9726 (1998).

${ }^{14}$ Q. Shi and E. Geva, J. Phys. Chem. A 107, 9059 (2003).

${ }^{15}$ J. A. Poulsen, G. Nyman, and P. J. Rossky, PNAS 102, 6709 (2005).

16 J. Liu and W. H. Miller, J.Chem. Phys. 125, 224104 (2006).

${ }^{17}$ M. Monteferrante, S. Bonella, and G. Ciccotti, Mol. Phys. 109, 3015 (2011)

${ }^{18}$ N. Makri, Phys. Chem. Chem. Phys. 13, 14442 (2011).

19 J. Liu and W. H. Miller, J. Chem. Phys. 134, 104102 (2011).

${ }^{20}$ J. Liu, J. Chem. Phys. 134, 194110 (2011).

${ }^{21}$ J. Liu, J. Chem. Phys. 140, 224107 (2014).

${ }^{22}$ J. Liu and Z. Zhang, J. Chem. Phys. 144, 034307 (2016).

${ }^{23}$ J. Liu, D. Li, and X. Liu, J. Chem. Phys. 145, 024103 (2016).

${ }^{24}$ J. Liu and W. H. Miller, J. Chem. Phys. 131, 074113 (2009).

${ }^{25}$ K. K. G. Smith, J. A. Poulsen, G. Nyman, and P. J. Rossky, J. Chem. Phys. 142, 244112 (2015).

${ }^{26}$ R. P. Feynman and H. Kleinert, Phys. Rev. A 34, 5080 (1986).

${ }^{27}$ S. Jang and G. A. Voth, J. Chem. Phys. 111, 2357 (1999).

${ }^{28}$ D. C. Marinica, M.-P. Gaigeot, and D. Borgis, Chem. Phys. Lett. 423, 390 (2006).

${ }^{29}$ P. Frantsuzov, A. Neumaier, and V. A. Mandelshtam, Chem. Phys. Lett. 381, 117 (2003).

${ }^{30}$ M. Basire, D. Borgis, and R. Vuilleumier, Phys. Chem. Chem. Phys. 15, 12591 (2013).

${ }^{31}$ J. Beutier, D. Borgis, R. Vuilleumier, and S. Bonella, J. Chem. Phys. 141, 084102 (2014).

${ }^{32}$ S. Blinnikov and R. Moessner, Astronomy and Astrophysics Supplement Series 130, 193 (1998).

${ }^{33} \mathrm{P}$. Hall, The bootstrap and Edgeworth expansion (Springer Science \& Business Media, 2013).

${ }^{34}$ M. Ceriotti, M. Parrinello, T. E. Markland, and D. E. Manolopoulos, J. Chem. Phys. 133, 124104 (2010).

${ }^{35}$ B. Leimkuhler and C. Matthews, App. Math. Res. Express 2013, 34 (2012).

${ }^{36}$ F. Mouhat, S. Sorella, R. Vuilleumier, A. M. Saitta, and M. Casula, J. Chem. Th. Comput. 13, 2400 (2017).

${ }^{37}$ J. Behler and M. Parrinello, Phys. Rev. Lett. 98 (2007), 10.1103/physrevlett.98.146401.

${ }^{38}$ J. Behler, Angew. Chem., Int. Ed. 56, 12828 (2017).

${ }^{39}$ A. P. Bartók, M. C. Payne, R. Kondor, and G. Csányi, Phys. Rev. Lett. 104 (2010), 10.1103/physrevlett.104.136403.

${ }^{40}$ A. P. Bartók, S. De, C. Poelking, N. Bernstein, J. R. Kermode, G. Csányi, and M. Ceriotti, Science Advances 3, e1701816 (2017).

${ }^{41}$ J. P. Alborzpour, D. P. Tew, and S. Habershon, J. Chem. Phys. 145, $174112(2016)$

${ }^{42}$ B. Leimkuhler and X. Shang, SIAM J. Sci. Comput. 38, A712 (2016).

${ }^{43}$ G. F. Reiter, J. Mayers, and P. Platzman, Phys. Rev. Lett. 89 (2002).

${ }^{44}$ I. R. Craig and D. E. Manolopoulos, J. Chem. Phys. 121, 3368 (2004).

${ }^{45}$ S. Habershon, D. E. Manolopoulos, T. E. Markland, and T. F.
Miller, Annu. Rev. Phys. Chem. 64, 387 (2013).

${ }^{46}$ M. Rossi, M. Ceriotti, and D. E. Manolopoulos, J. Chem. Phys. 140, 234116 (2014)

${ }^{47}$ J. Cao and G. A. Voth, J. Chem. Phys. 100, 5093 (1994).

${ }^{48}$ S. Jang and G. A. Voth, J. Chem. Phys. 111, 2371 (1999).

${ }^{49}$ M. Rossi, V. Kapil, and M. Ceriotti, J. Chem. Phys. 148, 102301 (2018), https://doi.org/10.1063/1.4990536.

${ }^{50}$ D. Li, X. Han, Y. Chai, C. Wang, Z. Zhang, Z. Chen, J. Liu, and J. Shao, J. Chem. Phys. 147, 184104 (2017), https://doi.org/10.1063/1.4996204.

${ }^{51}$ M. E. Tuckerman, Statistical Mechanics: Theory and Molecular Simulation (Oxford University Press, 2010) Chap. 12.4.1. 